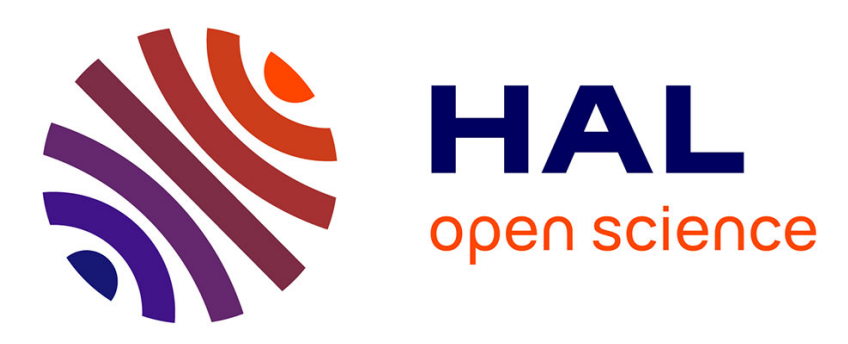

\title{
Evaluation of different turbulent combustion models based on tabulated chemistry using DNS of heterogeneous mixtures
}

Stephane Chevillard, Jean-Baptiste Michel, Cecile Pera, Julien Reveillon

\section{To cite this version:}

Stephane Chevillard, Jean-Baptiste Michel, Cecile Pera, Julien Reveillon. Evaluation of different turbulent combustion models based on tabulated chemistry using DNS of heterogeneous mixtures. Combustion Theory and Modelling, 2017, 21 (3), pp.440-465. 10.1080/13647830.2016.1247214 . hal01582158

\section{HAL Id: hal-01582158 \\ https://hal-ifp.archives-ouvertes.fr/hal-01582158}

Submitted on 13 Sep 2017

HAL is a multi-disciplinary open access archive for the deposit and dissemination of scientific research documents, whether they are published or not. The documents may come from teaching and research institutions in France or abroad, or from public or private research centers.
L'archive ouverte pluridisciplinaire $\mathbf{H A L}$, est destinée au dépôt et à la diffusion de documents scientifiques de niveau recherche, publiés ou non, émanant des établissements d'enseignement et de recherche français ou étrangers, des laboratoires publics ou privés. 


\section{HAL \\ Hat}

\section{Evaluation of different turbulent combustion models based on tabulated chemistry using DNS of heterogeneous mixtures}

Stephane Chevillard, Jean-Baptiste Michel, Cecile Pera, Julien Reveillon

\section{To cite this version:}

Stephane Chevillard, Jean-Baptiste Michel, Cecile Pera, Julien Reveillon. Evaluation of different turbulent combustion models based on tabulated chemistry using DNS of heterogeneous mixtures. Combustion Theory and Modelling, Taylor \& Francis, 2017, 21 (3), pp.440-465. .

\section{HAL Id: hal-01582158 https://hal-ifp.archives-ouvertes.fr/hal-01582158}

Submitted on 5 Sep 2017

HAL is a multi-disciplinary open access archive for the deposit and dissemination of scientific research documents, whether they are published or not. The documents may come from teaching and research institutions in France or abroad, or from public or private research centers.
L'archive ouverte pluridisciplinaire HAL, est destinée au dépôt et à la diffusion de documents scientifiques de niveau recherche, publiés ou non, émanant des établissements d'enseignement et de recherche français ou étrangers, des laboratoires publics ou privés. 
Combustion Theory and Modelling

Vol. 00, No. 00, June 2015, 1-20

\title{
RESEARCH ARTICLE
}

\section{Evaluation of different turbulent combustion models based on tabulated chemistry using DNS of heterogeneous mixtures}

\author{
Stephane Chevillard ${ }^{\mathrm{a}, \mathrm{b} *}$, Jean-Baptiste Michel $^{\mathrm{a}}$, Cecile Pera $^{\mathrm{a}, \mathrm{c}}$ and Julien Reveillon ${ }^{\mathrm{b}}$ \\ a IFP Energies nouvelles, 1 et 4 avenue de Bois-Préau, 92852 Rueil-Malmaison, France; \\ ${ }^{\mathrm{b}}$ CORIA, University and INSA of Rouen, avenue de l'Université, 76800 \\ Saint-Etienne-du-Rouvray, France \\ ${ }^{\mathrm{c}}$ Infineum UK Ltd, PO Box 1, Milton Hill, Abingdon OX13 6BB, England
}

(Received XX Month 2015; final version received XX Month 2015)

\begin{abstract}
This study used direct numerical simulations (DNSs) of combustion processes in turbulent heterogeneous mixtures for self-igniting partially-premixed configurations to assess the accuracy of partially-premixed turbulent combustion models that are based on the tabulation of chemistry progress in homogeneous reactors (HRs). DNS coupled with n-heptane/air detailed chemistry solving was considered as a reference result. Because the same detailed chemistry was used to generate the chemistry databases, the study was focused entirely on validating the modelling assumptions. Various HR-based tabulation models were tested: (1) the tabulated homogeneous reactor (THR) model, which is a direct exploitation of HR tabulation lacking any statistical information concerning mixture heterogeneity; (2) the presumed conditional moment $(\mathrm{PCM})$ model, which includes a limited statistical description of the mixture and/or of the combustion advancement; (3) approximated diffusion flame (ADF) models, which consider the heterogeneous turbulent reactor as either a unique diffusion flame (simple ADF model formulation) or as a collection of flamelets with different strain rates (ADF $\chi$ model). A priori response of the above mentioned models was compared with detailed chemistry DNS results. The main findings are as follows: (1) A direct use of HR tabulation (THR model) led to overly inaccurate results; (2) an assumed independence between mixture fraction and progress variable was responsible for most PCM modelling failures in the context of turbulent heterogeneous self-ignited combustion; (3) the presumed beta-function of the progress variable distribution is likely to fail because of the complexity of auto-ignition kinetics; (4) the best results were obtained with the ADF models; (5) a simple ADF formulation is preferable to $\mathrm{ADF} \chi$, which showed limitations in terms of accuracy concerning the distribution of the progress variable ;(6) all tested models provided an acceptable prediction of the auto ignition delays but only $\mathrm{ADF}$ and $\mathrm{ADF} \chi$ models are able to represent the temporal evolution of the progress variable.
\end{abstract}

Keywords: tabulated chemistry; autoignition; Diesel engine; PDF; flamelet

*Corresponding author. Email: stephane.chevillard@ifpen.fr 


\section{Nomenclature}

\section{English}

$\begin{array}{ll}c & \text { normalized progress variable } \\ \bar{c} & \text { mean (Reynolds-averaged) progress variable } \\ \overline{c^{\prime 2}} & \text { progress variable variance } \\ P & \text { probability density function } \\ \overline{S_{c}} & \text { progress variable segregation } \\ \widetilde{S_{Z}} & \text { mixture fraction unmixedness } \\ T & \text { temperature } \\ Y_{c} & \text { progress variable } \\ Y_{i} & \text { mass fraction of the } i^{\text {th }} \text { species } \\ Z & \text { mixture fraction } \\ \widetilde{Z} & \text { mean (Favre-averaged) mixture fraction } \\ \widetilde{Z^{\prime \prime 2}} & \text { mixture fraction variance }\end{array}$

\section{Greek}

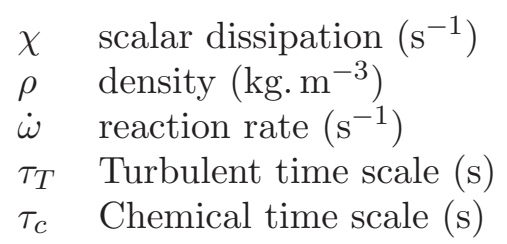

\section{Superscripts}

eq equilibrium

init initial conditions

HR homogeneous reactor

$\mathrm{u}$ fresh (unburned) gas

\section{Subscripts}

$\begin{array}{ll}\text { ADF } & \text { approximated diffusion flame } \\ \text { ADF } \chi & \text { approximated diffusion flame with a log-normal distribution of } \chi \\ \text { DNS } & \text { direct numerical simulation } \\ \max & \text { maximal } \\ \text { MR } & \text { most reactive } \\ \text { st } & \text { stoichiometric }\end{array}$

\section{Introduction}

The number of fundamental and applied studies on combustion under diesel engine conditions has increased dramatically in the past decades. Faced with ever greater demands for reduced fuel consumption and stringent pollutant emissions, this research is driven by the necessity to concurrently maintain the high thermal efficiency typical of compression-ignition engines [1]. Recent developments are increasingly reliant on computational fluid dynamics (CFD), which help evaluate the most promising strategies at the preliminary stages of engine development. In order to offer the precision needed to help meet future emission regulations for 
diesel engines, CFD codes require the introduction of a detailed chemistry. Only this level of description is able to correctly represent such characteristics as the evolution of autoignition delays, pollutant emissions, etc. for all engine operating conditions (fuel/air equivalence ratio, dilution, pressure, temperature).

Various strategies exist to introduce complex or detailed chemistry into CFD codes. Although it is possible to resolve detailed chemical schemes directly [2], this remains highly CPU-time intensive (about 21 hours on an 8-core machine for a 400-species mechanism of a typical diesel engine calculation) and thus not usable at industrial scale. In addition the turbulence-chemistry interaction has to be modelled. For this reason, several combustion models are based on the laminar diffusion flame equation introduced by Peters [3]. Among them, interesting results have been obtained by the representative interactive flamelet (RIF) model [4] in which a limited number of diffusion flamelets are calculated in a dedicated solver. At each time-step, the flamelet solutions are mapped onto the CFD-field. Because only a few diffusion flames are calculated, this model requires moderate CPU times; it was shown to give good results in Reynolds-Averaged Navier-Stokes (RANS) simulations of Diesel engines. From this model, the Eulerian Particle Flamelet Model was proposed [5] using Eulerian markers to represent more accurately the heterogeneity of scalar dissipation in the domain. Another solution is offered by the different tabulation strategies that have been proposed over the last two decades such as the Flame Prolongation of ILDM (FPI) [6] or the flame-generated manifold (FGM) [7], which rely on flamelet databases that are generated based on prototype combustion problems prior to any CFD computations. These models use a unique parameter to represent the chemical path (for any given thermodynamic condition): the normalized progress variable $(c)$, which ranges from zero in fresh gases to one in fully burned gases. Combustion characteristics are therefore assumed to be exclusively reliant on this progress variable, leading to a considerably reduced database. Using a similar notion of the progress variable, the TKI (Tabulated Kinetics for Ignition) model [8] proposes to generate a database of reactions rates to estimate the ignition of fuel/air mixtures. Coupled with the ECFM3Z model (3 Zones Extended Coherent Flame Model), this model has proven its capability to simulate several Diesel configurations as in [9]. In contrast with FPI and FGM, this model brings no information on the chemical composition and has to be coupled to another model to estimate the species concentrations. With different assumptions, the Flamelet Progress Variable (FPV) model proposed by Pierce and Moin [10] also introduces a progress variable instead of the scalar dissipation rate and has shown its ability to capture fine combustion effects such as partial extinctions [11]. However, this model is not suitable for assessing diffusion flame ignition. Finally, another possiblity is proposed by the Conditional Moment Closure (CMC) approach [12]. This model is featured by a general mathematical formalism which avoids the fomulation of any hypothesis about the topology of the reaction zone. As noted in [13], CMC has been applied to the simulation of autoigniting jets, but it was up to recently limited either to higher order closure with very simple kinetics or to more complex mechanisms using first-order closure due to the large CPU requirements.

The present work focused on FPI-like methods. The original FPI approach was based on the tabulation of a set of unstrained laminar premixed flames [6]. Following an appropriate reformulation of hypotheses concerning flame and flow physics, we were able to successfully apply this approach to partially-premixed [14] and non-premixed combustions [15] as well as to autoignition [16, 17] phenomena.

As far as auto-ignition is considered, the adaptation of the FPI approach included the replacement of unstrained laminar premixed flames by homogeneous reactors. The resulting look-up tables consisted of a collection of self-igniting premixed com- 
bustions in homogeneous reactors. The tabulation method is consequently called the tabulated homogeneous reactor (THR) method.

Apart from challenges related to the detailed chemistry, an appropriate treatment of the turbulence-chemistry interactions is necessary. Several turbulent combustion models have been proposed for RANS calculations with various levels of complexity depending on the statistical description of the mixture, which can be considered homogeneous [18] and is either modelled in several zones [8] or with a continuous probability density function of the mixture fraction and of the flame structure [12]. In the THR context, various models such as the presumed conditional moment (PCM) $[15,17]$ or the approximated diffusion flame (ADF) model [19] have been proposed in the literature.

Several studies have investigated the validation of existing THR modelling approaches either in academic geometries $[17,20,21]$ or in industrial contexts including diesel engines [9]. Such validations relied on comparisons between experimental data and RANS $[9,20]$ or large eddy simulation (LES) $[17,21]$ computations. Nevertheless, results are strongly dependent on the choice of the detailed chemistry mechanism and no obvious ranking on the prediction capability of these models can be drawn because it is not possible to determine whether the observed differences are due to modelling hypotheses or to the chemistry.

The present work provide a validation of the various turbulent models that are based on homogeneous reactor tabulations, excluding any possibly misleading influence of the chemistry description. For this purpose, we analysed direct numerical simulation (DNS) data coupled with detailed chemistry solving. The DNS configuration corresponded to a heterogeneous reactor in which various parameters (temperature, fuel-air equivalence ratio, etc.) were fully controlled. The same detailed chemistry was used to compute the DNS and to generate the chemistry database used in all the tested turbulent combustion models.

We first describe various turbulent combustion models that are based on the tabulation of HRs. Section 3 summarizes the DNS numerics and presents the turbulent heterogeneous reactor configuration. Comparisons between tabulation models and detailed chemistry DNS computations are discussed in Section 4. Finally, main conclusions are summarized.

\section{Description of the turbulent combustion models}

Various turbulent combustion models that are based on the tabulation of chemical reactions in HRs were compared. All of these models rely on the same look-up table for the chemical reaction rate description. Our chemistry database was generated from adiabatic HR at constant pressure computed with the same detailed mechanism [22] used in DNS solving (Section 3.2).

First, the global HR tabulation approach is introduced, then a brief introduction of each turbulent combustion model using such tabulations is presented.

HR tabulation is based on the same approach as FPI modelling [6, 16]. FPI-like tabulations [6] consist in relating all combustion quantities (species mass fraction, temperature, heat release, etc.) to a single progress variable $Y_{c}$, which quantifies the transition between fresh gases and fully burned products. In accordance with previously conducted FPI research $[14,15]$, we define $Y_{c}$ as follows:

$$
Y_{c}=Y_{C O}+Y_{C_{2}}
$$

For practical reasons, the progress variable is normalized and is denoted by $c$ as 
written below:

$$
c=\frac{Y_{c}}{Y_{c}^{e q}}
$$

where $Y_{c}^{e q}$ is the value of $Y_{c}$ when the thermodynamic equilibrium is reached in fully burned gases at constant pressure and enthalpy. This definition of the progress variable has been used in several past studies with n-heptane as fuel (see e.g. $[16,23,24])$. However, the presented models could be used with other definitions, as those of Bekdemir et al. [25, 26] and Egüz et al [27]. With Equation (2), the normalized progress variable grows monotonically from $c=0$ in fresh gases to a value of $c=1$ in the final combustion products. We verified this behaviour for all HRs in the present study.

The studied tabulation methods rely on the initial generation of a database of adiabatic HRs at constant pressure. The resulting mass fractions $Y_{i}$ are then stored as functions of $c$ and of the mixture fraction $Z$. Similarly, the reaction rate of the progress variable $\dot{\omega}_{Y_{c}}$ can also be stored.

The variety of initial thermodynamic conditions ${ }^{1}$ and compositions encountered in the DNS computations is taken into account by generating a database of fresh gas temperatures $T_{u}$ and fuel/air equivalence ratios related to the mixture fraction $Z$. For each HR initial temperature, $200 \mathrm{HR}$ computations reproduce the self-ignited combustion of mixtures covering the entire $Z$ domain $(0 \leq Z \leq 1)$. We performed these computations using the Senkin code from the Chemkin ${ }^{\circledR}$ II package [28].

Initial conditions of HRs can be defined as a linear function of $Z$ :

$$
\begin{gathered}
Y_{C_{7} H_{16}}^{\mathrm{init}}(Z)=Z, \\
Y_{O_{2}}^{\mathrm{init}}(Z)=(1-Z) Y_{O_{2}}^{\mathrm{air}}, \\
Y_{N_{2}}^{\text {init }}(Z)=(1-Z) Y_{N_{2}}^{\text {air }},
\end{gathered}
$$

where $Y_{\mathrm{O}_{2}}^{\text {air }}$ and $Y_{N_{2}}^{\text {air }}$ are the mass fraction of $\mathrm{O}_{2}$ and $\mathrm{N}_{2}$ in air, respectively. Concerning the fresh gas temperature, $T_{u}$ is assumed to be linear with the mixture fraction:

$$
T_{u}(Z)=(1-Z) T^{\text {air }}+Z T^{\text {fuel }} .
$$

Note that we also used Equation (4) for the initial DNS conditions (Section 3).

Finally the completed HR database contains all $Y_{i}$ and $\dot{\omega}_{Y_{c}}$ values stored at discrete points of a regular orthogonal table sampling as $(2000 \times 200)$ in $(c, Z)$ for each temperature condition. The domain of variation for $Z$ and $T_{u}(Z)$ in the look-up table covers all conditions encountered in the DNS computations (Table $1)$.

The HR database can be used with various turbulent combustion models, which are briefly described below. Model inputs and assumptions are summarized in Table 2 .

${ }^{1}$ For the DNS computations studied here, the pressure is constant (10 bar). Therefore, this coordinate can be omitted as a database input. 


\subsection{The THR approach}

The tabulated homogeneous reactor (THR) approach directly uses the HR look-up table value without any additional modelling to describe the heterogeneity influence. The THR model assumes that the global system reacts like a homogeneous reactor with the following characteristics: (1) its mixture fraction is equal to the (Favre-averaged) mean of the heterogeneous field and is denoted by $\widetilde{Z}=\overline{\rho Z} / \bar{\rho}$; (2) its normalized progress variable is equal to the mean of the heterogeneous field and is denoted by $\bar{c}$. Mean quantities are therefore directly extracted from the HR tabulation:

$$
\begin{gathered}
\widetilde{Y}_{i}=Y_{i}^{H R}(\widetilde{Z}, \bar{c}), \\
\widetilde{\dot{\omega}_{Y_{c}}}=\dot{\omega}_{Y_{c}}^{H R}(\widetilde{Z}, \bar{c}) .
\end{gathered}
$$

\subsection{The PCM approach}

Vervisch et al. [15] have proposed a more advanced model, called the presumed conditional moment (PCM), in which the influence of heterogeneity on reacting mixtures is taken into account through probability density functions (PDFs). Initially developed in the context of partially-premixed combustion [15], the PCM formalism was extended to turbulent self-ignited combustion by Galpin et al. [17], whose look-up table was generated from HRs. This section gives a brief description of the PCM model. For complete details we refer the reader to $[15,17]$.

PCM assumes that statistical behaviours can be described through a joint PDF of $c$ and $Z$. According to the HR tabulation-based PCM formalism [17], mean species mass fractions can be written as

$$
\tilde{Y}_{i}=\int_{0}^{Z_{\max }} \int_{0}^{1} Y_{i}^{\mathrm{HR}}(Z, c) \widetilde{P}(Z, c) d Z d c
$$

where $Y_{i}^{\mathrm{HR}}$ is the HR tabulated mass fraction of the $i^{\text {th }}$ species. Similarly, the mean progress variable reaction rate is reconstructed as

$$
\widetilde{\dot{\omega}_{Y_{c}}}=\int_{0}^{Z_{\max }} \int_{0}^{1} \dot{\omega}_{Y_{c}}^{\mathrm{HR}}(Z, c) \widetilde{P}(Z, c) d Z d c
$$

where $\dot{\omega}_{Y_{c}}^{\mathrm{HR}}$ is the progress variable reaction rate in tabulated HRs.

\section{Hypothesis 2.1:}

The PCM model [15] assumes that $c$ and $Z$ are two independent variables, permitting the joint PDF to be written as a product of two independent PDFs:

$$
\widetilde{P}(Z, c)=\widetilde{P}(Z) \bar{P}(c)
$$

Hypothesis 2.2: The mixture fraction $P D F, \widetilde{P}(Z)$, is presumed to be a $\beta$ function whose first and second moments are computed thanks to balance equations for $\widetilde{Z}$ and $\widetilde{Z^{\prime \prime 2}}$, respectively.

The mixture fraction variance $\widetilde{Z^{\prime \prime 2}}$ is conveniently normalized by its theoretical maximum to define the unmixedness, which evolves from Zmin (zero in pure air) 
to Zmax (unity in pure fuel) :

$$
\widetilde{S_{Z}}=\frac{\widetilde{Z^{\prime \prime 2}}}{\left(\widetilde{Z}-Z_{\min }\right)\left(Z_{\max }-\widetilde{Z}\right)} .
$$

Two options are available for the PCM approach. With the first option (referred to as the PCM1 model here), the fluctuations of the progress variable are neglected so that the progress variable $\mathrm{PDF}$ is $\bar{P}(c)=\delta(c-\bar{c})$, where $\delta$ is the Dirac delta function.

With the second option (referred to as the PCM2 model), the fluctuations of the progress variable are described with a presumed PDF according to the following assumption.

Hypothesis 2.3: The progress variable PDF, $\bar{P}(c)$, is presumed to be a $\beta$ function whose first and second moments are computed thanks to balance equations for $\bar{c}$ and $\overline{c^{\prime \prime 2}}$, respectively.

In general, reaction fluctuations are defined through a segregation factor:

$$
\overline{S_{c}}=\frac{\overline{c^{\prime 2}}}{\bar{c}(1-\bar{c})}
$$

Note that Equations (6) and (7) and Hypothesis 2.2 and 2.3 permit the THR approach to be seen as a zero-order PCM model in which $\widetilde{S_{Z}}$ and $\overline{S_{c}}$ are null, that is to say $\bar{P}(c)=\delta(c-\bar{c})$ and $\widetilde{P}(Z)=\delta(Z-\widetilde{Z})$.

\subsection{The ADF model}

The following briefly describes the approximated diffusion flames (ADF) model. Further details can be found in the article from Michel et al. [19].

The ADF model is based on the tabulation of $1 \mathrm{D}$ approximated diffusion flames [19] in a counterflow configuration. The generation of a database of HRs in which global reaction rate $\dot{\omega}_{Y_{c}}$ values can be stored as a function of $c$ and $Z$ serves as a starting point. Then, a library of approximated diffusion flames is built by solving Peters' flamelet equation [3] for the progress variable $Y_{c}$ :

$$
\frac{\partial Y_{c}}{\partial t}=\dot{\omega}_{Y_{c}}^{H R}(Z, c)+\chi\left(Z, \chi_{s t}\right) \frac{\partial^{2} Y_{c}}{\partial Z^{2}}
$$

where $\chi$ is the scalar dissipation rate, which is modelled using the classical expression for counterflow diffusion flames that incorporates the inverse of the error function $F(Z)$ [3]. The scalar dissipation rate $\chi$ can be related to only two parameters, namely $Z$ and the stoichiometric value of the scalar dissipation rate $\chi_{s t}$ following $\chi\left(Z, \chi_{s t}\right)=\chi_{s t} \times F(Z)$, for which the expression of the function $F(Z)$ can be found in [3].

Resolving the flamelet equation (Equation (11)) gives access to the evolution of $Y_{c}^{A D F}\left(Z, \chi_{s t}, t\right)$ and consequently to $c^{A D F}\left(Z, \chi_{s t}, t\right)$ (Equation (2)). Then, the mass fractions of all species $i$ at a given time $t$ are computed as

$$
Y_{i}^{A D F}\left(Z, \chi_{s t}, t\right)=Y_{i}^{H R}\left(Z, c\left(Z, \chi_{s t}, t\right)\right) .
$$

Because only one equation is solved with the flamelet formalism, very low CPU requirements are needed compared to the computation of any unsteady diffusion 
flame and regardless of the complexity of the detailed chemistry mechanism. This methodology allows in reproducing both the autoignition process and the steady flame structure of a laminar diffusion flame [19].

Once the approximated diffusion flames were computed, we performed an integration at each flamelet time using two PDFs: (1) According to Hypothesis 2.2, mixture fraction heterogeneities were modelled based on a classical $\beta$-function; (2) because we chose a PDF for $\chi_{s t}$, Equation (11) can be solved, and we can therefore deduce the PDF of $c$. Two options are available for ADF modelling. With the first option (referred to as ADF here), the 'standard' model represents $\chi_{s t}$ as a Dirac function at $\bar{\chi}_{s t}$. The second option (referred to as ADF $\chi$ ) presumes the statistical behaviour of $\chi_{s t}$ based on the following assumption:

Hypothesis 2.4: The PDF of the stoichiometric scalar dissipation $\bar{P}\left(\chi_{s t}\right)$ is presumed to be a log-normal function with a variance of $\sigma=1$ as proposed by Peters [3].

Note that, contrary to the PCM2 approach, the ADF model does not directly predict the shape of $\bar{P}(c)$. Nevertheless, $\bar{P}(c)$ can be estimated from tabulation inputs [19]. For the first-order ADF formulation, each mixture fraction has its own progress variable $c\left(Z, t, \overline{\chi_{\mathrm{st}}}\right)$ so that the conditional $\mathrm{PDF}$ of the progress variable can be written as follows:

$$
P_{A D F}(c \mid Z)=\delta\left(c-c_{\mathrm{ADF}}\left(Z, t, \overline{\chi_{\mathrm{st}}}\right)\right) .
$$

Integration over the mixture fraction domain allows us to determine the progress variable PDF:

$$
\widetilde{P_{A D F}}(c)=\int_{0}^{Z_{\max }} \widetilde{P}(Z) \delta\left(c-c_{\mathrm{ADF}}\left(Z, t, \overline{\chi_{\mathrm{st}}}\right)\right) d Z
$$

For the ADF $\chi$ model, we can also derive the expression of $P(c \mid Z)$, as detailed in Michel et al. [20]:

$$
P_{A D F \chi}(c \mid Z)=\int_{0}^{\infty} \delta\left(c-c_{\mathrm{ADF} \chi}\left(Z, t, \overline{\chi_{\mathrm{st}}}\right)\right) \bar{P}\left(\chi_{\mathrm{st}}\right) d \chi_{\mathrm{st}} .
$$

A similar integration over $Z$ provides the Favre-averaged PDF of $c$ :

$$
\widetilde{P_{A D F \chi}}(c)=\int_{0}^{Z_{\max }} \int_{0}^{\infty} \delta\left(c-c_{\mathrm{ADF} \chi}\left(Z, t, \overline{\chi_{\mathrm{st}}}\right)\right) \bar{P}\left(\chi_{\mathrm{st}}\right) d \chi_{\mathrm{st}} \widetilde{P}(Z) d Z
$$

Michel et al. [29] showed that the conditioned probability given by Equation 15 permits retrieving the experimental shape and the mixture fraction dependence of the Cabra et al. experiment [30].

In the frame of the use these models in CFD codes, the table is generated in two steps. In a first step, parameters of interest such as mean mass fractions or the mean reaction rate are computed by integration of the local and instantaneous values of the approximated flamelets - functions of $\left(Z, \chi_{s t}, t\right)$ - at each flamelet time over PDFs of $Z$ and $\chi_{s t}: \widetilde{Y_{i}(t)}=\int_{0}^{Z_{\max }} \int_{0}^{\infty} Y_{i}^{A D F}\left(Z, \chi_{s t}, t\right) d Z d \chi_{s t}$. This operation can also be done for the averaged progress variable $\widetilde{Y}_{c}$. In a second step, the resulting mean quantities are tabulated as functions of $\widetilde{Y}_{c}$ using the bijectivity between the flamelet time and $\widetilde{Y}_{c}$. 


\section{Numerical procedure and computational parameters}

\subsection{DNS numerics}

We used the DNS code Asphodele [31] (which is based on a dilatable low-Mach approach detailed in [32]) and applied the sixth order Padé scheme from [33] to compute spatial derivatives on a regular mesh. Time integration was carried out with a third order explicit Runge-Kutta scheme with a minimal data storage method [34]. In addition to classical Navier-Stokes equations (mass, momentum, and energy equations), mass fractions were transported for all species of the detailed mechanism.

Patel's mechanism [22] for n-heptane/air mixtures (29 species and 52 reactions) was solved by coupling the DNS solver with the Chemkin VODE solver [31]. Patel's mechanism [22] has been extensively validated through comparisons with reference detailed chemistry schemes and with experimental data [22, 35]. It has been proven that the mechanism correctly captures both low- and high-temperature reaction characteristics, including the cool-flame phenomenon and the negative temperature coefficient (NTC) ${ }^{1}$ auto-ignition behaviour [35].

\subsection{DNS configuration}

The present DNS configuration corresponds to the self-ignited combustion of a heterogeneous mixture (Figure 1) in a spatially decaying homogeneous and isotropic turbulence.

Because we used DNS in combination with detailed chemistry solving, the required CPU time was a major limitation. Sreedhara and Lakshmisha [36] have previously discussed the suitability of a two-dimensional configuration to study autoignition in heterogeneous mixtures. They observed similar auto-ignition behaviours for both 2D- and 3D-computations with a four-step chemistry. Recently, 3D DNS computations with a detailed chemistry [37] have confirmed 2D-observations whose first ignition spots originate at locations where $\chi$ is low and $Z$ is close to the most reactive value.

The present work aims to validate turbulent combustion models based on HR tabulation in heterogeneous reactors. A 2D-heterogeneous reactor can be considered an improvement compared with the usual test cases in homogeneous reactors [17]. Although differences between 2D- and 3D-auto-ignition synopses are to be expected, a turbulent heterogeneous reactor remains a relevant test case to validate model hypotheses. Nevertheless, further studies should investigate 3D DNS results to confirm our observations.

We initialized the turbulence velocity field by specifying a Passot-Pouquet [38] energy spectrum in Fourier space. The integral length scale was $L_{t}=0.34 \mathrm{~mm}$ and the initial fluctuating velocity was $u^{\prime}=1 \mathrm{~m} \mathrm{~s}^{-1}$ leading to a $R e_{T}=22$. Turbulence was isotropically decaying, and there was no initial mean flow.

A square domain of $4 \mathrm{~mm}$ side length $\left(11.7 L_{t}\right)$ with a uniform grid resolution of $\Delta_{x}=14 \mu \mathrm{m}$ served as the basis for the 2D computations. The initial ambient pressure was $p=10$ bar. Note that the chosen pressure value allowed us to study NTC phenomena even though it is well below the levels found in actual diesel engines. Each 2D simulation case (Section 3.3) was completed in approximately 100 hours using a parallel MPI domain partition on 64 processors of a SGE Altix ICE 8200 cluster.

${ }^{1}$ Within the NTC region, the auto-ignition delay of a purely gaseous and homogeneous mixture increases with temperature. 
Auto-ignition occurred in a partially premixed environment created from initially prescribed heterogeneities (Figure 1). Initial mixture fields were generated with a prescribed amplitude and characteristic size (equal, in this work, to the integral length scale) based on a spectral procedure described in Reveillon [39]. The heterogeneity distribution was initially Gaussian over the entire domain. An example of the initial field is shown in Figure 1.

DNS configurations can be characterized looking at the characteristic time scales. First of all, an interesting comparison concerns the auto-ignition delay with the integral time scale. In this work, the auto-ignition time scale can be estimated between 1000 and $2000 \mu s$ depending on the configuration and the turbulent time scale close to $340 \mu \mathrm{s}$. This means that auto-ignition occurs during the mixing process. This turbulent time scale can also be compared to a chemical characteristic time representative of the maximal reaction rate of the homogeneous reactor (as $\left.\left(T_{a d}-T_{i n i}\right) / \max \left(\frac{d T}{d t}\right)\right)$, leading to a definition of a Damköhler number, around 60 at stoichiometry and under 4 for the richest area. Finally, the chemical time scale can also be compared to a turbulent time scale of the Kolmogorov scales, which is here close to $80 \mu \mathrm{s}$. This comparison leads to the definition of a Karlovitz number, which is included between 0.075 and 1 in this work. Such values show that turbulence and combustion are intricate processes in the chosen configuration.

\subsection{DNS database}

Table 1 summarizes the multiple initial conditions used in our study. Two main parameters were investigated: the initial temperature and the initial mixture. Cases denoted by $M$ - focused on mixture issues, whereas cases denoted by $T$ - investigated temperature influence. Except for Cases -TvXXX-YYY (Table 1), the chosen temperature applied to both $T^{\text {air }}$ and $T^{\text {fuel }}$, leading to a uniform initial $T_{u}(Z)$ (Equation (4)) denoted by -TuXXX in Table 1.

To build a reference without any NTC or cool flame phenomena, we chose an initial uniform high temperature of $T^{\text {init }}=1100 \mathrm{~K}$. The first variation (Cases M-SzXX-Z05-TU1100) concerned unmixedness: values for $\widetilde{S_{Z}}$ were 0.1 (nearly homogeneous), 0.2 (intermediate unmixedness) or 0.6 (high unmixedness).

In order to investigate the influence of saturation-limited evaporation (in real diesel engines, droplet evaporation is limited by local vapor conditions), we studied various maximum mixture fractions $\left(Z_{\max }\right)$ in Cases M-Sz06-ZXX-TU1100 while keeping a constant high unmixedness $\left(\widetilde{S_{Z}}=0.6\right)$.

We then investigated uniform intermediate and low temperatures (T-Sz06-Z01TUXXX). Initial temperatures were chosen based on homogeneous autoignition results [35]. Representative of a high temperature behaviour, $T^{\text {init }}=1100 \mathrm{~K} \mathrm{elim-}$ inates any NTC or cool flame phenomenon. We chose $T^{\text {init }}=800 \mathrm{~K}$ because, at 10 bar, the temperature is below the NTC region. Eventually, we purposely chose $T^{\text {init }}=950 \mathrm{~K}$ because it is within the NTC region.

Finally, we investigated the temperature's dependence on the mixture fraction in order to mimic the cooling effect caused by fuel droplet evaporation in actual diesel engines (temperature is assumed to linearly evolve with $Z$ ). Mixing temperatures varied for a given mixture fraction range of $0<Z<0.5$ ( $T^{\text {air }}$ and $T^{\text {fuel }}$ were set at different initial values (Equation (4)), with fuel colder than air). A uniform temperature of $T^{\text {init }}=800 \mathrm{~K}$ served as a reference. We then investigated two temperature slopes for $T_{u}(Z)$ : The first slope considered $T^{\text {fuel }}=650 \mathrm{~K}$ and $T^{\text {air }}=$ 950 K (Case T-Sz06-Z01-Tv650-950); it was within the NTC range [35]. The second slope considered $T^{\text {fuel }}=500 \mathrm{~K}$ and $T^{\text {air }}=1100 \mathrm{~K}$ (Case T-Sz06-Z01-Tv500-1100); it spread on each side of the NTC area. 
The relatively large number of cases allows the investigation of the models answers over various conditions which could be encountered in a Diesel Spray. Results section will only focus on few configurations which give specific information on models behaviour.

\subsection{DNS post-processing for model validation}

The comparisons between DNS and models are a priori comparisons, excluding therefore errors linked to numerics and other models. Several past studies have been devoted to the a priori validation of other turbulent combustion models using either DNS results [40] or experimental results [41, 42]. In the present work, the comparison with DNS results has been retained as it excludes potential errors from the chemistry modelling from the comparisons.

The same detailed chemistry for n-heptane/air mixtures as Patel et al. [22] is used for both DNS and tabulation models. Consequently, observed errors are only linked to modelling formulations and no additional error could be attributed to the the kinetics selection.

DNS data are post-processed to obtain relevant inputs for the tabulation models: the mean and variance of the mixture fraction and the mean and variance of the progress variable, etc. (Table 2). The DNS results (reaction rates, for instance) were then compared with the model predictions, which yielded a priori validations (Section 4).

Model input parameters (Table 2) were computed from DNS data using either Reynolds-averaging $(\bar{Q})$ or Favre- (mass weighting-) averaging $(\widetilde{Q})$. One main parameter was the mixture fraction $Z$, which characterizes local mixing. As far as a detailed chemistry is concerned, various definitions are possible [43]. In the present work, we computed the $Z$ definition from detailed-chemistry DNS thanks to carbon atom conservation. The progress variable $\left(Y_{c}\right)$ was computed with Equation (1) and normalized (c) according to Equation (2). To define the progress variable and the mixture fraction unmixedness, we estimated the variance (Reynolds or Favre formulation) through Equations (9) and (10).

The last input parameter in Table 2 is the mean stoichiometric scalar dissipation $\left(\overline{\chi_{\mathrm{st}}}\right)$, that is to say the average of $\left(\chi \mid Z_{\mathrm{st}}\right)$. This parameter was computed by averaging the value of $\chi$ in cells where $Z=Z_{\text {st }}$.

Interestingly, a potential comparison of DNS results with the RIF or conditional moment closure (CMC) models should consider the evolution of a laminar diffusion flamelet ${ }^{1}$ integrated over a probability density function of the mixture fraction (which is a beta-function). As a consequence, we would expect these models to behave similarly to ADF, attributing the differences to errors between approximated and exact laminar diffusion flamelets.

\section{Results}

\subsection{General observations}

Figure 2 shows the general character of the turbulent simulations in terms of progress variable and mixture fraction. Due to turbulence, n-heptane and air mix first (1.2 ms). At $2.1 \mathrm{~ms}$, several ignition spots develop, and global thermal runaway occurs soon after $(2.3 \mathrm{~ms})$.

\footnotetext{
${ }^{1}$ As we only consider one cell without transport phenomena, the first order CMC equation simplifies into a flamelet equation.
} 
It has previously been demonstrated that auto-ignition usually occurs away from stoichiometry at a "most reactive mixture fraction" denoted by $Z_{\mathrm{MR}}$, which can be identified through HR auto-ignition [44]. Considering the dependence of temperature on the mixture fraction imposed in Case T-Sz06-Z01-Tv500-1100, homogeneous auto-ignitions suggest that the earliest auto-ignition should be observed with rich mixture compositions at $Z_{\mathrm{MR}} \sim 0.25$.

Figure 3 shows scatterplots of the progress variable over the simulation domain at the three key instants selected in Figure $2(1.2 \mathrm{~ms}, 2.1$, and $2.3 \mathrm{~ms})$. During the pre-ignition phase (Figure 3(a)), chemistry progress first occurred at a rich mixture fraction $(Z=0.24)$ close to $Z_{\mathrm{MR}} \sim 0.25$. As chemistry progressed, the burning spots grew larger while expanding toward leaner mixtures (Figure 2 at $2.1 \mathrm{~ms}$ ). Therefore, the most reactive regions shifted toward stoichiometry (Figure $3(\mathrm{~b})$ ). Eventually, the reaction zones merged into each other (Figure 2 at $2.3 \mathrm{~ms}$ ), and the progress variable grew toward unity everywhere (Figure 3(c)).

We validated the turbulent combustion models (Table 2 ) based on a priori analyses of the DNS results. Mean mass fractions and reaction rates of all major and radical species were compared. We observed similar behaviours for all of these quantities. For brevity's sake, only mean reaction rates of the progress variable and $\mathrm{CO}_{2}$ and $\mathrm{OH}$ mean mass fractions are presented here. The mean reaction rate of the progress variable $\dot{\bar{\omega}}_{Y_{c}}$ allowed us to predict autoignition characteristics (delay and heat release). $\mathrm{CO}_{2}$ is representative of the behaviour of the main combustion products; $O H$ illustrates highly reactive radical species.

\subsection{Influence of the mixture fraction unmixedness}

We investigated model response to a variation in unmixedness based on Cases M-SzXX-Z05-TU1100 (Table 1), for which $\widetilde{S_{Z}}=0.1$ (nearly homogeneous), 0.2 (intermediate unmixedness) or 0.6 (high unmixedness). The higher the unmixedness, the weaker $\widetilde{\dot{\omega}_{Y_{c}}}$ (omitted here) because very rich and very lean mixtures have a low reactivity.

Figure 4 shows the mean reaction rate of the progress variable $\widetilde{\dot{\omega}_{Y_{c}}}$ during autoignition progression for Case M-Sz02-Z05-TU1100 $\left(\widetilde{S_{Z}}=0.2\right)$. The THR model clearly overestimated $\widetilde{\dot{\omega}_{Y_{c}}}$ (Figure 4) whatever the unmixedness (Sz01Z05TU1100 and Sz06Z05TU1100 are omitted here). Contrary to the main combustion products $\left(\mathrm{Y}_{\mathrm{CO}_{2}}\right.$ in Figure 5), intermediate species $\left(Y_{\mathrm{OH}}\right.$ in Figure 5) were inadequately predicted by the THR model. We observed similar results irrespective of the unmixedness (Cases Sz01-Z05-TU1100 and Sz06-Z05-TU1100). These results show that, even for low heterogeneities, a homogeneous mixture cannot be assumed in tabulation modelling.

It is insufficient to only take the mixture fraction heterogeneities into account because the PCM1 model also overestimated $\widetilde{\dot{\omega}_{Y_{c}}}$ (Figure 4 ). This behaviour is consistent with previous results by Michel et al. [20], which were further confirmed in the LES context by Galpin et al. [17]. Michel et al. [20] attributed the overestimation of the PCM1 mean reaction rate to Hypothesis 2.1. We discuss this assumption in Section 4.5.

The PCM2 approach considerably improved predictions of both minor species evolutions $\left(\widetilde{Y_{O H}}\right.$ in Figure 5$)$ and of the mean progress variable reaction rate $\widetilde{\dot{\omega}_{Y_{c}}}$

in Figure 4). Nevertheless, although PCM2 correctly predicted $\widetilde{Y_{O H}}$ and $\widetilde{\dot{\omega}_{Y_{c}}}$ for the early auto-ignition step $\left(\widetilde{Y}_{c}<0.02\right.$ in Figure 4$)$, it slightly overestimated them for high progress variable values.

The mean progress variable reaction rate $\widetilde{\dot{\omega}_{Y_{c}}}$ and all species mass fractions were 
well reproduced by both ADF models. Note that, for all M-SzXX-Z05-TU1100 cases, $\mathrm{ADF}$ and $\mathrm{ADF}_{\chi}$ provided very similar results (Figure 4).

To understand the discrepancies between DNS results and PCM models, we investigated mixture fraction and progress variable PDFs. For this purpose, distributions were reconstructed from DNS data and compared to the presumed PDFs of the PCM models (Hypothesis 2.2 and 2.3) for case M-Sz02-Z05-TU1100 for two representative combustion processes (Figure 6). The first distribution, at $\widetilde{Y}_{c}=0.015$, showed good agreement between DNS and PCM2 modelling (Figure 4). The second distribution, at $\widetilde{Y}_{c}=0.045$, showed some deviation (Figure 4).

Corresponding mixture fraction and progress variable PDFs are shown in Figure 6 . We were able to confirm the assumed $\beta$-function for the mixture fraction in PCM and ADF models (Hypothesis 2.2) for all auto-ignition processes (Figure 6(a) and (b)). The $\beta$-function provided a very good approximation of the mixture fraction distribution and is consequently not responsible for the discrepancies between DNS results and PCM predictions.

We then investigated Hypothesis 2.3 by comparing $\bar{P}(c)$ values extracted from DNS results with the corresponding $\beta$-function (Figure 6(c) and (d)). For the early step of the autoignition $\left(\widetilde{Y}_{c}=0.015\right), \bar{P}(c)$ was well reproduced by the $\beta$-function assumed for PCM2 (Figure 6(c)). Agreement was less obvious for the later stages $\left(\widetilde{Y}_{c}=0.045\right)$. Figure $6(\mathrm{~d})$ shows that the $c$ distribution "froze" near $c \sim 0.6$. However, this stagnation is transitory because, evidently, $c=1$ at the end of the fuel oxidation process (Figure 7). Due to the presence of very rich mixture fractions, equilibrium is reached very slowly because some pyrolyse kinetics occur just after complete consumption of deficient $\mathrm{O}_{2}$. Consequently, the progress variable seems to be "frozen" for an intermediate value of $c$ (Figure 7). The bimodal distribution of $c$ with a stagnation at an intermediate value cannot be reproduced with the $\beta$-function assumed for the PCM2 model. In contrast, ADF and ADF $\chi$ models do not presume the shape of $\bar{P}(c)$ but reconstruct it from precomputed diffusion flames [19]. Figure 6(c) and (d) shows that both ADF and ADF $\chi$ models predicted the progress variable distribution very well, including the progress variable pause.

For all M-SzXX-Z05-TU1100 Cases, ADF and ADF $\chi$ models gave very similar results. Nevertheless, discrepancies may appear, for example in cases where the mean mixture fraction evolves.

\subsection{Influence of mean mixture fraction}

We investigated the influence of the mean mixture fraction $\widetilde{Z}$ on HR tabulationbased model responses through Cases M-Sz06-ZXX-TU1100 (Table 1) for which $\widetilde{Z}$ $=0.1,0.25$ or 0.5 . The higher the mean mixture fraction, the weaker $\widetilde{\dot{\omega}_{Y_{c}}}$ but also the smaller the final $\widetilde{Y}_{c}$ (omitted here). In fact, very rich mixtures led to a lower $C O$ and $\mathrm{CO}_{2}$ production and consequently to lower $Y_{c}$ values (Equation (1)). Because the progress variable reaction rate is related to $Y_{c}$ production, richer mixtures imposed a weaker $\widetilde{\dot{\omega}_{Y_{c}}}$. Nevertheless, the normalized reaction rate $\widetilde{\dot{\omega}_{Y_{c}}} / \widetilde{Y_{e q}}$ was of the same order for any $\widetilde{Z}$ variation. All tabulation models were able to reproduce this trend. However, as for unmixedness variation cases (Section 4.2), THR, PCM1, and PCM2 models were unable to correctly estimate the mean reaction rate, neither for the shape nor for the order of magnitude (Figure 8).

Contrary to the previous examples, Case M-Sz06-Z01-Tu1100 (low $\widetilde{Z}$ ) showed differences between both ADF approaches (Figure 8). Surprisingly, ADF $\chi$ was less predictive than the simple ADF formulation.

$\mathrm{ADF}$ and $\mathrm{ADF} \chi$ differ in their scalar dissipation PDF modelling (Table 2). We 
studied the PDF of $\chi_{s t}$ to understand the discrepancy in results between ADF $\chi$ modelling and ADF prediction (which offered better agreement with DNS results). Figure 9 plots $\bar{P}\left(\chi_{s t}\right)$ at $\widetilde{Y}_{c}=0.12$, the instant of highest discrepancy between ADF $\chi$ and DNS data (Figure 8). We observed that the log-normal distribution was a good approximation of $\bar{P}\left(\chi_{s t}\right)$, and very good agreement existed between the log-normal distribution and DNS results whatever the case from Table 1. Consequently, the log-normal assumption (Hypothesis 2.4) cannot be the cause of the ADF $\chi$ error.

Then, we investigated the conditional $\operatorname{PDF} \bar{P}\left(c \mid Z^{*}\right)$ for the same time of $\tilde{Y}_{c}$ $=0.12$ (Figure 10). We chose $Z^{*}=0.13$ because this value corresponded to the most likely mixture fraction in the heterogeneous reactor at $\widetilde{Y}_{c}=0.12$ for Case M-Sz06-Z01-Tu1100. Similarly to Figure 6(d), $\bar{P}\left(c \mid Z^{*}\right)$ extracted from DNS results presented a bimodal distribution (Figure 10). The ADF $\chi$ model also deduced a bimodal distribution but its second peak was slightly staggered toward $c=1$. Yet, toward the burned gases $(c \sim 0.95)$, the reaction rate was smaller than for the actual peak of $\bar{P}_{D N S}\left(c \mid Z^{*}\right)$ occurring for $0.8<c<0.95$ (Figure 10). Consequently, the reaction rate retrieved by the ADF $\chi$ model, corresponding to the integration of the product of $\dot{\omega}_{H R}\left(c \mid Z^{*}\right)$ and $\bar{P}\left(c \mid Z^{*}\right)$, was weaker than the DNS results (Figure $8)$.

The main reasons explaining the slight shift of the second peak of the $\overline{P_{A D F \chi}}\left(c \mid Z^{*}\right)$ function towards burnt gases (which leads to a non negligible variation of the mean reaction rate) are the strong assumptions used to generate the $A D F \chi$ table: we assume indeed that the heterogeneous reactor can be represented by a collection of several independent flamelets, each one being characterized by

- a $\chi_{\text {st }}$ value constant in time

- a weight given by the log normal $\mathrm{P}\left(\chi_{\text {st }}\right)$ function (which should not be responsible for the error as presented previously)

- the same starting time (from the fresh gases state)

- no interaction with other flamelets

It is clear that assumptions concerning the independence of flamelets as well as the constant value of $\chi_{s t}$ are very strong and do not correspond to what was observed by analysing the DNS fields: clearly, for a considered element of flame; the scalar dissipation evolves with time and the different structures can interact.

The basic formulation of $\mathrm{ADF}$, however, presumed $\bar{P}\left(c \mid Z^{*}\right)$ as a Dirac on $c \sim 0.9$ (Figure 10) so that the integrated reaction rate was larger for $\mathrm{ADF}$ than for $\mathrm{ADF} \chi$ and fitted the DNS results better (Figure 8).

This result illustrates that, even if ADF $\chi$ includes more physical details than $\mathrm{ADF}$ and is able to retrieve the bimodal shapes of the $\bar{P}\left(c \mid Z^{*}\right)$ functions and their evolutions with the mixture fraction (not shown here), the model's sensitivity to the $\dot{\omega}_{H R}\left(c \mid Z^{*}\right)$ evolution makes it difficult to improve the final result.

\subsection{Influence of fresh gas temperature}

Sensitivity to the initial temperature was performed to test the tabulation-based models within the NTC range: $800 \mathrm{~K}$ (T-Sz06-Z01-TU800) corresponded to a low temperature case below the NTC region; $950 \mathrm{~K}$ (T-Sz06-Z01-TU950) was within the NTC region; $1100 \mathrm{~K}$ (T-Sz06-Z01-TU1100) corresponded to a high temperature case far from the NTC area.

Figure 11(a) shows a $\widetilde{\dot{\omega}_{Y_{c}}}$ comparison between DNS results and the various models for $T^{\text {init }}=950 \mathrm{~K}$ (within the NTC range).

Similarly to Section 4.2 (and for the same reasons), THR and PCM1 models greatly overestimated $\widetilde{\dot{\omega}_{Y_{c}}}$ (Figure $11(\mathrm{a})$ ) and thus inadequately predicted species 
mass fractions (omitted here).

PCM2 predictions were debased within the NTC, highly overestimating the reaction rate (Figure 11(a)). Indeed, near the NTC region, a cool flame chemistry may occur, leading to a temporary stagnation of the progress variable for an intermediate value of $c$. Moreover, the bimodal $\bar{P}(c)$ shape cannot be reproduced by a $\beta$-function if the second peak is not centred on either 0 or 1 .

The simple form of the ADF model gave satisfactory results (Figure 11(a)). With the ADF $\chi$ model, $\widetilde{\dot{\omega}_{Y_{c}}}$ was generally underestimated for $T^{\text {init }}=950 \mathrm{~K}$ (Figure 11(a)). A deeper analysis revealed similar mechanisms as described in Section 4.3: the second peak of the conditional $\operatorname{PDF} \bar{P}\left(c \mid Z^{*}\right)$ was shifted toward the burned gases, which increased the weight on the low reactivity area (omitted here).

The specific chemistry involved in the NTC phenomenon did not modify the quality of the ADF model predictions, whereas cool flames jeopardized the PCM $\beta$-function for the $c$ distribution (Hypothesis 2.3).

\subsection{Influence of the mixing temperature profile}

Finally, we considered more realistic configurations, involving an initial temperature dependency on the local mixture fraction. This last case mimicked two-phase flow conditions, in which evaporating fuel droplets locally modify the gas temperature and the equivalence ratio. This cooling effect leads to low temperatures in the rich mixture region.

Figure 11(b) illustrates the model response for Case T-Sz06-Z01-Tv650-950. THR and PCM models continually overestimated $\widetilde{\dot{\omega}_{Y_{c}}}$. The incorrect shape of the presumed $\bar{P}(c)$ and the homogeneous mixture hypothesis for THR were again responsible for these inadequate predictions (Figure 11(b)). Moreover, ADF and ADF $\chi$ models gave satisfactory results for $\widetilde{\dot{\omega}_{Y_{c}}}$ (Figure $11(\mathrm{~b})$ ) and for all species mass fractions (omitted here).

Finally, based on Case T-Sz06-Z01-Tv650-950, we identified the errors introduced by the PCM2 model assumptions (Hypothesis 2.1 to 2.3) through an analysis of the DNS results. Figure 12 presents $\overline{\dot{\omega}_{Y_{c}}}$ for DNS data and for various hypotheses of the PCM2 model.

First, we verified the basic formalism of the PCM2 approach (Equation (7)). For this purpose, we computed $\widetilde{\dot{\omega}_{Y_{c}}}$ and introduced the exact $\widetilde{P}(Z, c)$ extracted from the DNS analysis in Equation (7). Figure 12 shows that the PCM2 approach was able to reproduce $\widetilde{\dot{\omega}_{Y_{c}}}$ very well, provided that the exact $\widetilde{P_{\mathrm{DNS}}}(Z, c)$ was used to represent the joint fluctuations of the mixture fraction and the progress variable. The PCM2 formalism led to a weak error of about $3 \%$ of the total error (PCM2 modelling error), which confirms that it is possible to integrate a homogeneous reactor reaction rate to reproduce the complex auto-ignition of a turbulent heterogeneous reactor.

We then evaluated Hypothesis 2.1 (mutual independence of $Z$ and $c$ ) by computing $\widetilde{\dot{\omega}_{Y_{c}}}$ and introducing the decoupled product $\widetilde{P}_{\mathrm{DNS}}(Z) \bar{P}_{\mathrm{DNS}}(c)$. Thus, the exact mixture fraction and progress variable distribution of the DNS results was used to account for $Z$ and $c$ fluctuations, assuming their mutual independence. Figure 12 shows that this hypothesis introduced a major error of about $70 \%$ of the total error (complete PCM2 modelling error, which confirms observations made in previous studies [42] where conditional probabilities $\bar{P}\left(c \mid Z^{*}\right)$ were strongly dependent on the mixture fraction.

Hypothesis 2.3 ( $\beta$-function for the $c$ distribution) was verified by replacing the exact progress variable distribution with $\beta(c)$ (whereas the $Z$ distribution was 
computed thanks to $\left.\widetilde{P}_{\mathrm{DNS}}(Z)\right)$. Figure 6 shows that this approximation may fail but the question remains, "How large is the error?" The deficiency of Hypothesis 2.3 is confirmed in Figure 12. Use of a $\beta$-PDF to take the progress variable fluctuations into account led to an error of about $23 \%$ of the total PCM2 error.

Finally, Hypothesis 2.2 ( $\beta$-function for the $Z$ distribution) was tested by replacing the exact mixture fraction distribution with $\beta(Z)$. Figure 6 shows that this assumption added only a minor error (about $2 \%$ of the total PCM2 error), which is consistent with $\widetilde{P}(Z)$ observations (Figure 6).

Our analysis confirms that the assumption of statistical independence of the mixture fraction and the progress variable lead to the failure of PCM models in the context of self-ignited combustion. ADF models that do not include such a hypothesis therefore offer better predictions of the reaction rate for any condition.

\subsection{Analyses of the auto-ignition process}

In the previous comparison, the links between the models hypotheses and their impact on the progress variable reaction rates have been underlined. However, it is difficult to draw conclusions in terms of auto-ignition delay predictions as it mainly depends on the values of the reaction rate at very low values of the progress variable. For this reason, the results of the previous comparisons were stored as functions $\widetilde{\dot{\omega}_{Y_{c}}}\left(\widetilde{Y}_{c}\right)$ for all cases and all models, and the following equation was integrated:

$$
\frac{d \widetilde{Y}_{c}}{d t}=\widetilde{\dot{\omega}_{Y_{c}}}\left(\widetilde{Y}_{c}\right)
$$

By integrating this equation, the evolution of $\widetilde{Y}_{c}(t)$ could be compared for DNS and all models. From this result a value of the auto-ignition delay could be calculated. In this work, it was chosen to define the AI delay as the time needed to reach half of the maximum value of the normalised progress variable. The criterion choice has no impact on the below drawn conclusions (not shown here).

Figure 13 shows an example of this methodology for two different cases. Figure 13 (a) illustrates the averaged progress variable evolution for M-Sz06-Z05-Tu1100 case in which auto-ignition occurs in one step. In this kind of configuration all models satisfactorily reproduced the auto-ignition process. Auto-ignition delay was also correctly estimated : $34 \%$ error for the worst case (here PCM2 model). This error is the same order of magnitude as the uncertainties related to n-heptane chemical scheme. Figure 13 (b) illustrates the averaged progress variable evolution for MSz06-Z05-Tu1100 case in which the global auto-ignition occurs in several steps. For such conditions, only $\mathrm{ADF}$ and $\mathrm{ADF}_{\chi}$ models satisfactorily represents the autoignition process. For PCM models, first ignition step is well estimated but these models hardly reproduced the chemistry slowdown between $c=0.3$ and $c=0.45$.

Models were finally evaluated on the complete data base through the estimation of the relative error on Auto Ignition delay as defined previously. Such a quantity only inform on the overall auto-ignition process and set apart the actual temporal evolutions. Figure 14 shows the relative models errors on AI delay. The simplest model (THR) is the least accurate model regarding AI delay, its error can reach up to $100 \%$ for configurations in which the temperature varies with mixture fraction. PCM1 and PCM2 models provide satisfactory assessments of the AI delays as the maximum relative error is around $60 \%$. Auto Ignition delays predicted by $\mathrm{ADF}$ and $\mathrm{ADF}_{\chi}$ models are very close to each other and closer to DNS data excepted for T-Sz-Z01-Tv650-950 case. In this case ADF $\chi$ model under estimates AI delay. The probable reason is that using a log-normal distribution for the stoichio- 
metric scalar dissipation rate, $\mathrm{ADF}_{\chi}$ gives a non negligible weight to low strained flamelets (which have shortest delays). This should theoretically be compensated by flamelets with high scalar dissipation rates values (which have higher ignition delays). However depending on the conditions (here air and fuel temperatures), the increase of the ignition delay with the scalar dissipation rate as well as the maximal value of $\chi_{s t}$ allowing ignition can differ from a real diffusion flame. Therefore, if the PDF of scalar dissipation rate gives an important weight to such values of $\chi_{s t}$, it can lead to errors on the reaction rate or on the ignition delay. Finally, Table 3 presents the average values of the relative error on the AI delay prediction on the complete database for all models. This table shows that all models are able to give an acceptable estimation of the AI delay. The best estimation is provided by ADF model which is fully coherent with the observations made previously.

\section{Conclusions}

This study examined self-ignited combustion in turbulent heterogeneous reactors through DNS coupled with detailed chemistry solving in order to validate turbulent combustion models based on tabulated chemistry. The common principle was to take detailed chemistry effects into account through an off-line generation of chemistry look-up tables based on auto-igniting homogeneous reactors. Various tabulation models were tested: direct use of a tabulated homogeneous reactor (THR); presumed conditional moment (PCM) models, which used a mixture fraction $\beta$-PDF (PCM1 and PCM2) and a progress variable $\beta$-PDF (PCM2) to take mixture heterogeneity into account; approximated diffusion flame (ADF) models, which were based on coupling the THR method, the flamelet equation of Peters, and the PCM approaches.

We systematically tested the accuracy of such tabulation models in the context of turbulent heterogeneous self-ignited combustion. A priori response of tabulated models was compared with fully detailed chemistry DNS computations. Because DNS computations and tabulated chemistry models used the same detailed chemistry scheme, we were able to focus exclusively on validating the modelling assumptions.

The tabulated chemistry approach based on homogeneous reactors was shown to very precisely retrieve the DNS behaviours, given perfect modelling of the joint probability density function $\bar{P}(c \mid Z)$ that was directly deduced from the DNS results. Consequently, observed discrepancies between models and averaged DNS results were essentially due to assumptions concerning the flame structure and the different probability density function estimations; the discrepancies did not result from errors caused by the tabulated chemistry approach itself.

Key observations concerning the model validations are summarized in the following:

(1) Direct use of the homogeneous reactor tabulation (THR) method led to overly inaccurate results. This behaviour could be extended to all models, assuming a complete homogeneity of the RANS computational cell.

(2) Considering only the mixture fraction heterogeneity, (PCM1) was insufficient to improve the model prediction.

(3) Statistic mixing behaviours can be taken into account by using a $\beta$-function of the mixture fraction PDF.

(4) The presumed $\beta$-function for the progress variable distribution may fail if the kinetics include some stagnation of $c$ (cool flame or fuel pyrolyse).

(5) An analysis of the conditional probability density functions $\bar{P}(c \mid Z)$ showed 
that the assumption of independence between the mixture fraction and the progress variable is inexact; this assumption is proven to be responsible for the observed discrepancies with the PCM models

(6) The log-normal distribution offered a proper approximation of the scalar dissipation distribution.

(7) Best results were obtained with the ADF models for all DNS configurations; the simple ADF formulation was preferable.

(8) The most advanced ADF model formulation (ADF $\chi$ ) was the only model able to retrieve the bimodal shapes of $\bar{P}(c \mid Z)$ function with their dependence on mixture fraction. In contrast, in a few cases, this model underpredicted the average reaction rate.

(9) The models ability to correctly estimate the AI delay was investigated. All tested models provided an acceptable prediction of the AI delays but only $\mathrm{ADF}$ and $\mathrm{ADF}_{\chi}$ models were able to represent the temporal evolution of the progress variable.

Our results illustrate coherence of the model assumptions (diffusion flamelet structure, beta PDF of the mixture fraction, no statistical independence of $c$ and $Z$ ) that are also the basis of several other combustion models. Indeed, in similar configurations, both RIF and CMC models assume, as ADF, a diffusion flame structure averaged at each time over a probability density function of the mixture fraction. We would therefore expect these models to give results comparable to those obtained with ADF.

\section{Acknowledgements}

This work was granted access to the HPC resources of CINES under allocation c20132b6672 made by GENCI (Grand Equipement National de Calcul Intensif).

\section{References}

[1] J. Dec, Advanced compression-ignition engines - understanding the in-cylinder processes, Proc. Combust. Inst. 32 (2009), pp. 2727-2742.

[2] C. Naik, K. Puduppakkam, and E. Meeks, Simulation and analysis of in-cylinder soot formation in a low temperature combustion Diesel engine using a detailed reaction mechanism, SAE Paper 2013-01-1565 (2013).

[3] N. Peters, Turbulent Combustion Cambridge University Press, Cambridge, 2000.

[4] H. Pitsch, H. Barths, and N. Peters, Three-Dimensional Modeling of NOx and Soot Formation in DIDiesel Engines Using Detailed Chemistry Based on the Interactive Flamelet Approach, SAE Paper 962059 (1996).

[5] H. Barths, C. Hasse, G. Bikas, and N. Peters, Simulation of combustion in direct injection Diesel engines using a Eulerian particle flamelet model, Proc. Combust. Inst. 28 (2000), pp. 1161-1168.

[6] O. Gicquel, N. Darabiha, and D. Thevenin, Laminar premixed hydrogen/air counterflow flame simulations using flame prolongation of ILDM with differential diffusion, Proc. Combust. Inst. 28 (2000), pp. 2419-2425.

[7] J.A. van Oijen, F.A. Lammers, and L.P.H. de Goey, Modeling of complex premixed burner systems by using flamelet-generated manifolds, Combust. Flame 127 (2001), pp. 2124-2134.

[8] O. Colin and A. Benkenida, The 3-zones extended coherent flame model (ECFM3Z) for computing premixed/diffusion combustion, Oil Gas Sci. Technol. 59 (2004), pp. 593-609.

[9] L. Martinez, J.B. Michel, S. Jay, and O. Colin, Evaluation of different tabulation techniques dedicated to the prediction of the combustion and pollutants emissions on a Diesel engine with $3 D C F D$, SAE Paper 2013-01-1093 (2013).

[10] C. Pierce and P. Moin, Progress-variable approach for large-eddy simulation of non-premixed turbulent combustion, J. Fluid Mech. 504 (2004), pp. 73-97.

[11] M. Ihme and H. Pitsch, Prediction of extinction and reignition in nonpremixed turbulent flames using a flamelet/progress variable model: 2. Application in LES of Sandia flames D and E, Combust. Flame 155 (2008), pp. 90 - 107.

[12] A. Klimenko and R. Bilger, Conditional moment closure for turbulent combustion, Prog. Energy Combust. Sci. 25 (1999), pp. 595-687.

[13] G.D. Paola, I.S. Kim, and E. Mastorakos, Second-Order Conditional Moment Closure Simulations of 
Autoignition of an n-heptane Plume in a Turbulent Coflow of Heated Air, Flow Turbul. Combust. 82 (2008), pp. $455-475$.

[14] B. Fiorina, R. Baron, O. Gicquel, D. Thevenin, S. Carpentier, and N. Darabiha, Modelling nonadiabatic partially premixed flames using flame-prolongation of ILDM, Combust. Theory Model. 7 (2003), pp. 449-470.

[15] L. Vervisch, R. Hauguel, P. Domingo, and M. Rullaud, Three facets of turbulent combustion modelling: $D N S$ of premixed $V$-flame, LES of lifted nonpremixed flame and RANS of jet-flame, J. Turbulence 5 (2004).

[16] C. Pera, O. Colin, and S. Jay, Development of a FPI detailed chemistry tabulation methodology for internal combustion engines, Oil Gas Sci. Technol. 64 (2009), pp. 243-258.

[17] J. Galpin, C. Angelberger, A. Naudin, and L. Vervisch, Large-eddy simulation of H2/air auto-ignition using tabulated detailed chemistry, J. Turbulence 9 (2008).

[18] S. Singh, R. Reitz, and M. Musculus, Comparison of the characteristic time (CTC), representative interactive flamelet (RIF), and direct integration with detailed chemistry combustion models against optical diagnostic data for multi-mode combustion in a heavy-duty DI Diesel engine, SAE Paper 2006-01-0055 (2006).

[19] J.B. Michel, O. Colin, and D. Veynante, Modeling ignition and chemical structure of partially premixed turbulent flames using tabulated chemistry, Combust. Flame 152 (2008), pp. 80-99.

[20] J.B. Michel, O. Colin, and D. Veynante, Comparison of differing formulations of the PCM model by their application to the simulation of an auto-igniting H2/air jet, Flow Turbulence Combust. 83 (2009), pp. 33-60.

[21] J. Tillou, J.B. Michel, C. Angelberger, and D. Veynante, Assessing LES models based on tabulated chemistry for the simulation of Diesel spray combustion, Combust. Flame 161 (2014), pp. 525-540.

[22] A. Patel, S. Kong, and R. Reitz, Development and validation of a reduced reaction mechanism for HCCI engine simulations, SAE Paper 2004-01-0558 (2004).

[23] J.B. Michel and O. Colin, A tabulated diffusion flame model applied to diesel engine simulation, Int. J. Eng. R. 15 (2014), pp. 346-369.

[24] S. Jay and O. Colin, A variable volume approach of tabulated detailed chemistry and its applications to multidimensional engine simulations, Proc. Combust. Inst. 33 (2011), pp. 3065-3072.

[25] C. Bekdemir, L.M.T. Somers, L.P.H. de Goey, J. Tillou, and C. Angelberger, Predicting diesel combustion characteristics with Large-Eddy Simulations including tabulated chemical kinetics, Proc. Combust. Inst. 34 (2013), pp. 3067-3074.

[26] C. Bekdemir, B. Somers, and P. de Goey, DNS with detailed and tabulated chemistry of engine relevant igniting systems, Combust. Flame 161 (2014), pp. 201-221.

[27] U. Eguz, S. Ayyapureddi, C. Bekdemir, B. Somers, and P. de Goey, Manifold resolution study of the FGM method for an igniting diesel spray, Fuel 113 (2013), pp. 228-238.

[28] R. Kee, F. Rupley, and J. Miller, Chemkin II: a Fortran chemical kinetics package for the analysis of gas-phase chemical kinetics, Tech. Rep. SAND89-8009B, Sandia National Laboratories, 1989.

[29] J.B. Michel, O. Colin, C. Angelberger, and D. Veynante, Using the tabulated diffusion flamelet model ADF-PCM to simulate a lifted methane-air jet flame, Combust. Flame 156 (2009), pp. 1318-1331.

[30] R. Cabra, T. Myhrvold, J. Chen, R. Dibble, A. Karpetis, and R. Barlow, Simultaneous laser RamanRayleigh-LIF measurements and numerical modeling results of a lifted turbulent H2/N2 jet flame in a vitiated coflow, Proc. Combust. Inst. 29 (2002), pp. 1881-1888.

[31] J. Reveillon, C. Pera, and Z. Bouali, Examples of the potential of DNS for the undestanding of reactive multiphase flows, Int. J. Spray Combust. Dyn. 3 (2011), pp. 65-94.

[32] M. Chauvy, B. Delhom, J. Reveillon, and F. Demoulin, Flame/Wall Interactions: Laminar Study of Unburnt HC Formation, Flow Turbulence Combust. 84 (2010), pp. 369-396.

[33] S. Lele, Compact finite difference schemes with spectral like resolution, J. Comput. Phys. 103 (1992), pp. $16-42$.

[34] A. Wray, Minimal storage time-advancement schemes for spectral methods, Tech. Rep., Center for Turbulence Research, Stanford University, 1990.

[35] Z. Bouali, C. Pera, and J. Reveillon, Numerical analysis of the influence of two-phase flow mass and heat transfer on n-heptane autoignition, Combust. Flame 159 (2012), pp. 65-94.

[36] S. Sreedhara and N. Lakshmisha, Autoignition in a non-premixed medium: DNS studies on the effects of three-dimensional turbulence, Proc. Combust. Inst. 29 (2002), pp. 2051-2059.

[37] G. Borghesi, E. Mastorakos, and R. Cant, Complex chemistry DNS of n-heptane spray autoignition at high pressure and intermediate temperature conditions, Combust. Flame 160 (2013), pp. 1254-1275.

[38] T. Passot and A. Pouquet, Numerical simulation of homogeneous flows in the turbulent regime, J. Fluid Mech. 181 (1987), pp. 441-466.

[39] J. Reveillon, Numerical procedures to generate and to visualize flow fields from analytical or experimental statistics: turbulent velocity, fluctuating scalars and variable density sprays, Flow Visualization Image Proc. 12 (2005), pp. 1-19.

[40] M. Ihme, C.M. Cha, and H. Pitsch, Prediction of local extinction and re-ignition effects in nonpremixed turbulent combustion using a flamelet/progress variable approach, Proc. Combust. Inst. 30 (2005), pp. 793-800.

[41] W.J.S. Ramaekers, J.A. van Oijen, and L. de Goey, A priori testing of Flamelet Generated Manifolds for turbulent partially Premixed Methane / Air flames, Flow Turbulence Combust. 84 (2010), pp. 439-458.

[42] R.D. Meester, B. Naud, and B. Merci, A priori investigation of PDF modeling assumptions for a turbulent swirling bluff body flame ('SM1'), Combust. Flame 159 (2012), pp. 3353-3357.

[43] H. Pitsch and N. Peters, A consistent flamelet formulation for non-premixed combustion considering differential diffusion effects, Combust. Flame 114 (1998), pp. 26-40.

[44] E. Mastorakos, Ignition of turbulent non-premixed flames, Prog. Energy Combust. Sci. 35 (2009), pp. $57-97$. 
Table 1. List of DNS parametric studies. M-Cases refer to mixing variations, whereas T-cases investigate temperature influence.

\begin{tabular}{lccccc}
\hline Case & $\widetilde{S_{Z}}[-]$ & $\widetilde{Z}[-]$ & $Z_{\max }$ & Temp. & $T^{\text {init }}[\mathrm{K}]$ \\
\hline M-Sz01-Z05-Tu1100 & 0.1 & 0.5 & 1 & uniform & 1100 \\
M-Sz02-Z05-Tu1100 & 0.2 & 0.5 & 1 & uniform & 1100 \\
M-Sz06-Z05-Tu1100 & 0.6 & 0.5 & 1 & uniform & 1100 \\
\hline M-Sz06-Z025-Tu1100 & 0.6 & 0.25 & 0.5 & uniform & 1100 \\
M-Sz06-Z01-Tu1100 & 0.6 & 0.1 & 0.2 & uniform & 1100 \\
\hline T-Sz06-Z01-Tu1100 & 0.6 & 0.1 & 0.5 & uniform & 1100 \\
T-Sz06-Z01-Tu950 & 0.6 & 0.1 & 0.5 & uniform & 950 \\
T-Sz06-Z01-Tu800 & 0.6 & 0.1 & 0.5 & uniform & 800 \\
\hline T-Sz06-Z01-Tv650-950 & 0.6 & 0.1 & 0.5 & variable & $800 \pm 150$ \\
T-Sz06-Z01-Tv500-1100 & 0.6 & 0.1 & 0.5 & variable & $800 \pm 300$ \\
\hline
\end{tabular}

Table 2. Main modelling parameters of the THR [16], PCM [15, 17], and ADF models [19]. undef. indicates that the input parameter is not defined for the considered model.

\begin{tabular}{lccccc}
\hline Model & THR & PCM1 & PCM2 & ADF & ADF $\chi$ \\
\hline Inputs & $\bar{c}, \widetilde{Z}$ & $\bar{c}, \widetilde{Z}, \widetilde{S_{Z}}$ & $\bar{c}, \overline{S_{c}}, \widetilde{Z}, \widetilde{S_{Z}}$ & $\widetilde{Y_{c}}, \overline{\chi_{\mathrm{st}}}, \widetilde{Z}, \widetilde{S_{Z}}$ & $\widetilde{Y_{c}}, \overline{\chi_{\mathrm{st}}}, \widetilde{Z}, \widetilde{S_{Z}}$ \\
\hline$\tilde{P}(Z)$ & $\delta(Z-\widetilde{Z})$ & $\beta\left(\widetilde{Z}, \widetilde{S_{Z}}\right)$ & $\beta\left(\widetilde{Z}, \widetilde{S_{Z}}\right)$ & $\beta\left(\widetilde{Z}, \widetilde{S_{Z}}\right)$ & $\beta\left(\widetilde{Z}, \widetilde{S_{Z}}\right)$ \\
$\bar{P}(c)$ & $\delta(c-\bar{c})$ & $\delta(c-\bar{c})$ & $\beta\left(\bar{c}, \overline{S_{c}}\right)$ & Eq. $(14)$ & Eq. $(16)$ \\
$\bar{P}(c \mid Z)$ & $\bar{P}(c)$ & $\bar{P}(c)$ & $\bar{P}(c)$ & Eq. $(13)$ & Eq. $(15)$ \\
$\bar{P}\left(\chi_{\text {st }}\right)$ & undef. & undef. & undef. & $\delta\left(\chi_{\text {st }}-\overline{\chi_{\text {st }}}\right)$ & $P_{\log _{N}}$ \\
\hline
\end{tabular}

Table 3. Averaged absolute error in AI delay prediction for the complete data base

\begin{tabular}{lccccc}
\hline Model & THR & PCM1 & PCM2 & ADF & ADF $\chi$ \\
\hline Averaged absolute relative error & $47 \%$ & $31 \%$ & $27 \%$ & $16 \%$ & $20 \%$ \\
\hline
\end{tabular}

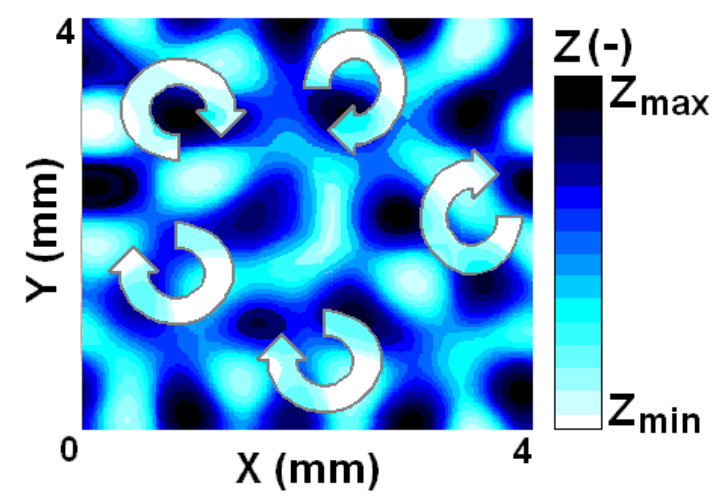

Figure 1. Initial conditions of the DNS configuration of an auto-igniting heterogeneous reactor. 

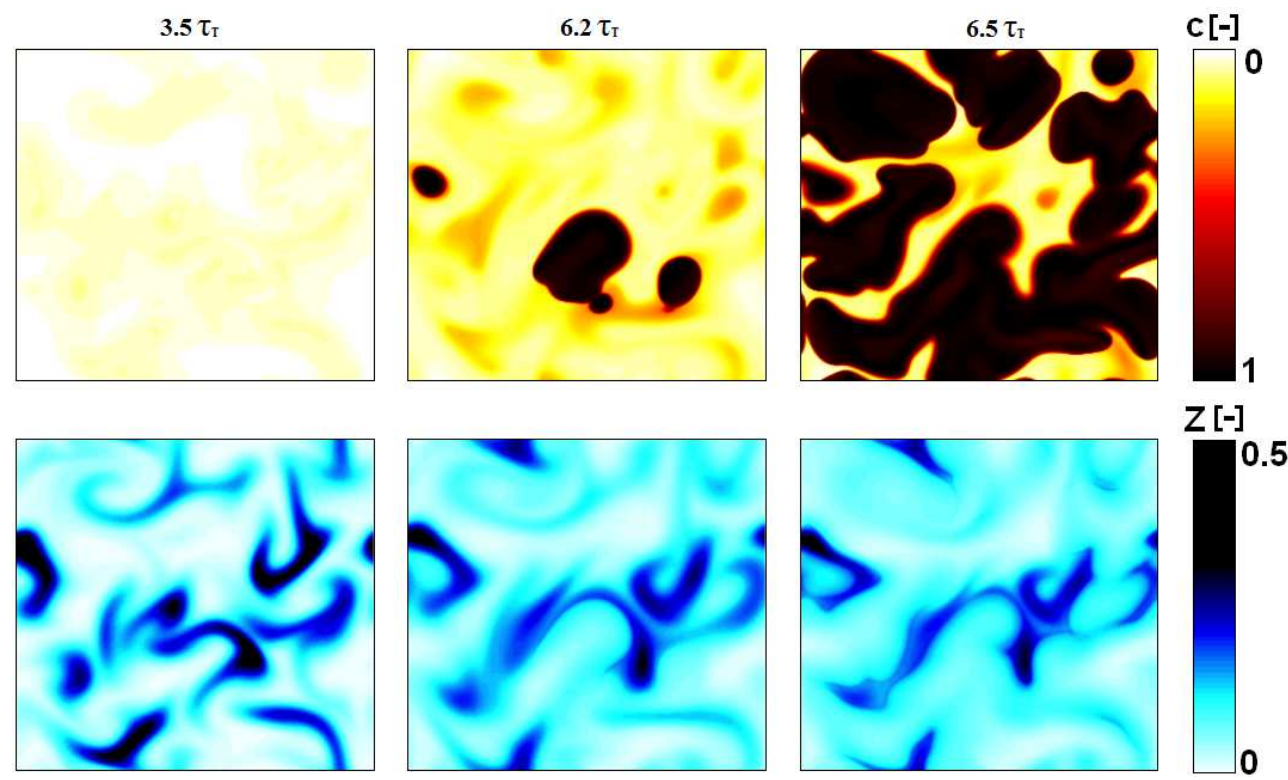

Figure 2. Autoignition of an n-heptane/air mixture: contour plots of the progress variable (top) and the mixture fraction (bottom). Data shown correspond to Case T-Sz06-Z01-Tv500-1100 in Table 1.

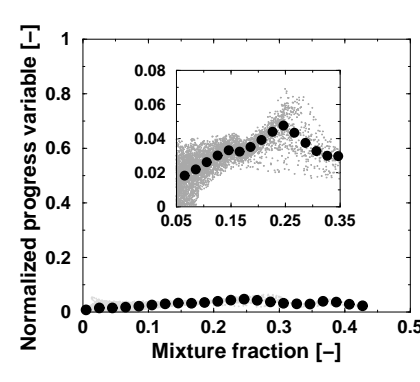

(a) $t=3.5 \tau_{t}$

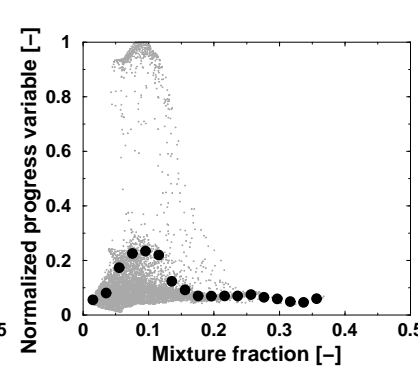

(b) $t=6.2 \tau_{t}$

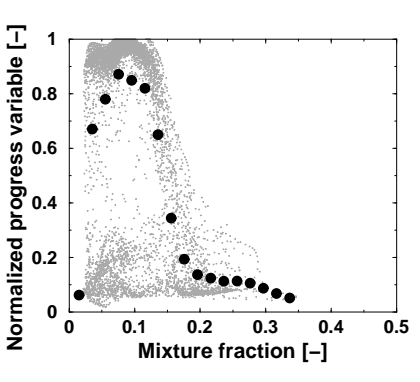

(c) $t=6.8 \tau_{t}$

Figure 3. Scatterplots (dots) of the normalized progress variable, $c$, against the mixture fraction, $Z$. Large black circles represent the conditional average progress variable $\left(\overline{c \mid Z^{*}}\right)$. Case T-Sz06-Z01Tv500-1100 in Table 1. 


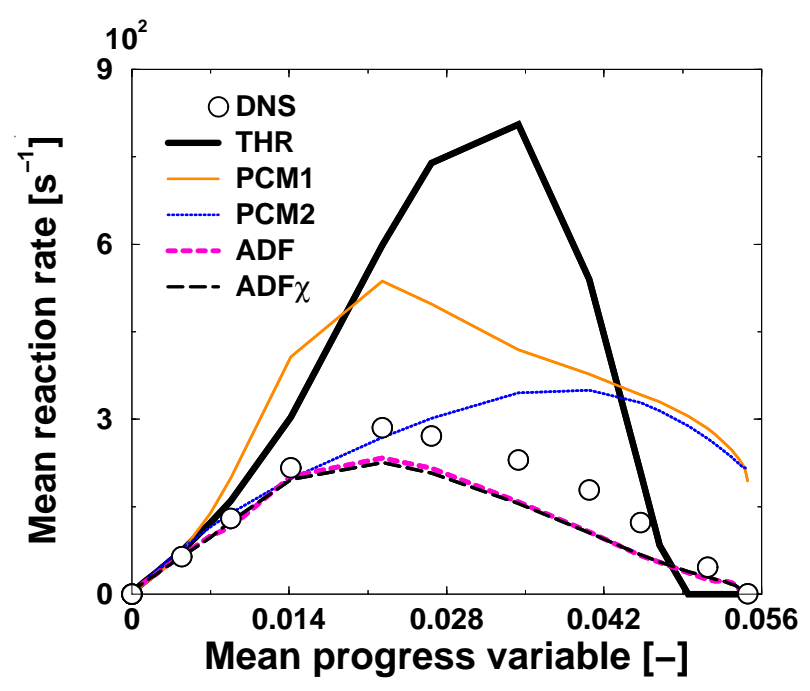

Figure 4. Evolution of the mean reaction rate of the progress variable $\left(\widetilde{\dot{\omega}_{Y_{c}}}\right)$ with the mean progress variable. Comparison between DNS results and tabulation models (Table 2). Case M-Sz02-Z05-Tu1100 (medium unmixedness).

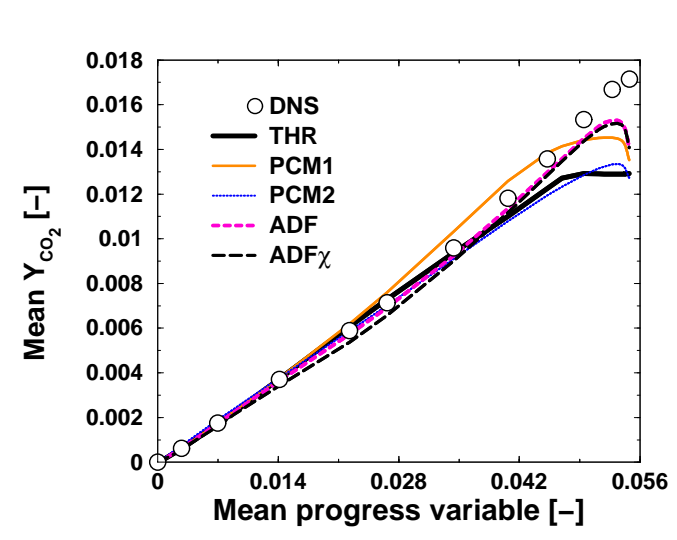

(a) $\widetilde{Y_{C O_{2}}}$

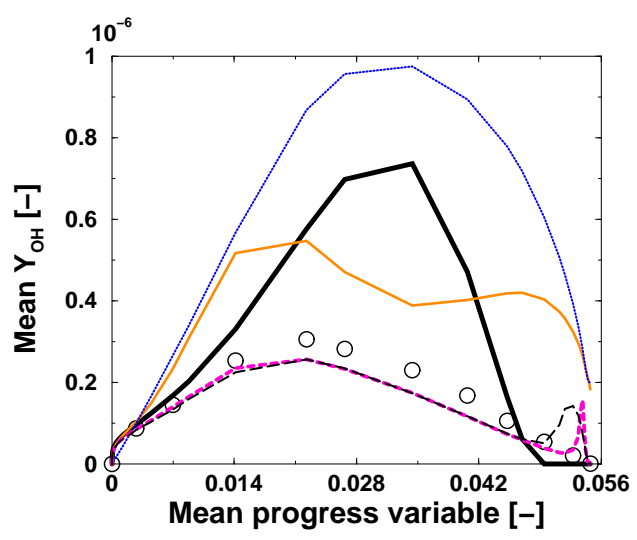

(b) $\widetilde{Y_{O H}}$

Figure 5. $\mathrm{CO}_{2}$ and $\mathrm{OH}$ mean mass fractions. Comparison between DNS results and tabulation models (Table 2). Case M-Sz02-Z05-Tu1100 (medium unmixedness). 


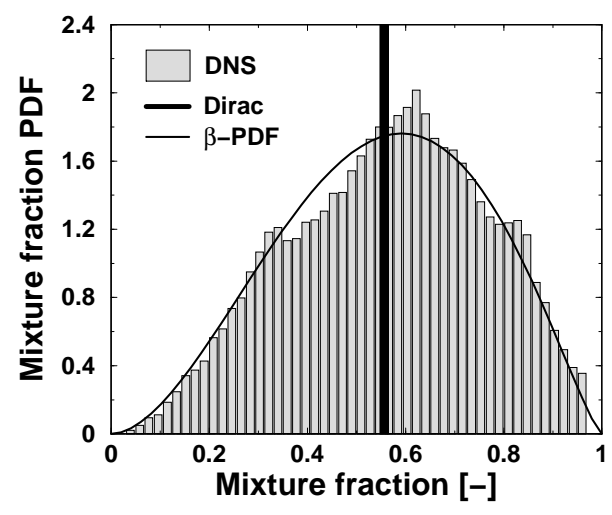

(a) $\widetilde{P}(Z)$ at $\widetilde{Y_{c}}=0.015$

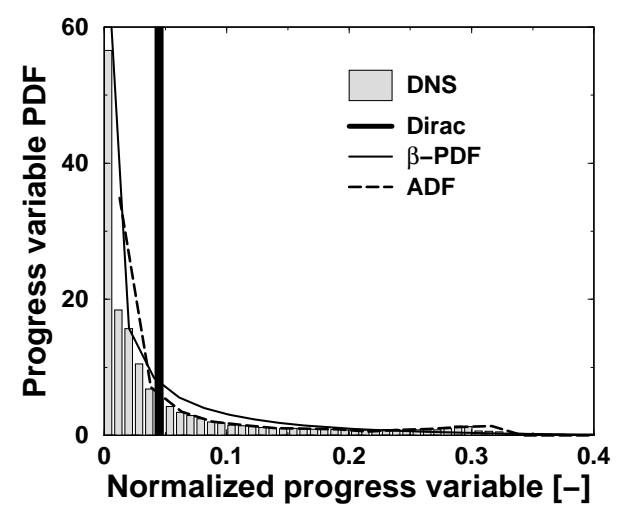

(c) $\bar{P}(c)$ at $\widetilde{Y_{c}}=0.015$

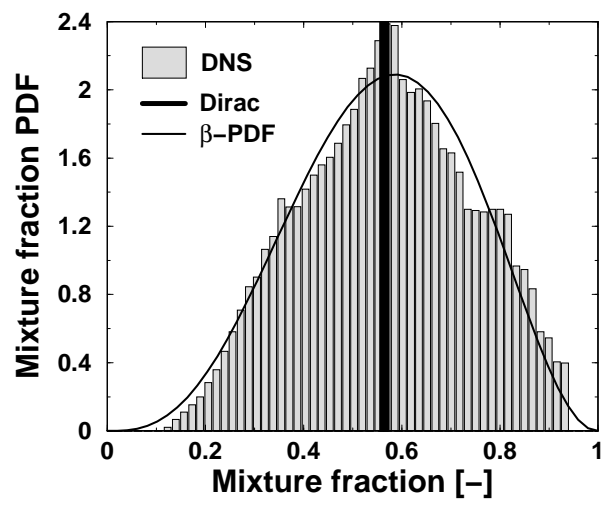

(b) $\widetilde{P}(Z)$ at $\widetilde{Y_{c}}=0.045$

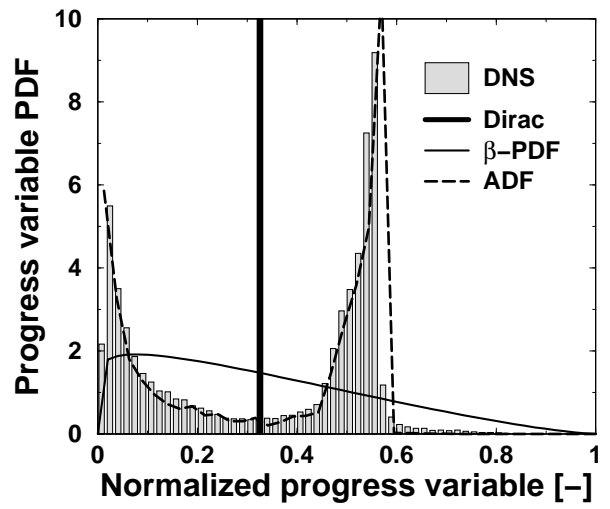

(d) $\bar{P}(c)$ at $\widetilde{Y_{c}}=0.045$

Figure 6. Probability density function of the mixture fraction (top) and of the normalized progress variable (bottom) at two combustion advancements, $\widetilde{Y_{c}}=0.015$ (left) and $\widetilde{Y_{c}}=0.045$ (right). Comparison between DNS data distributions and the presumed PDFs according to model (Table 2), Case M-Sz02-Z05-Tu1100.

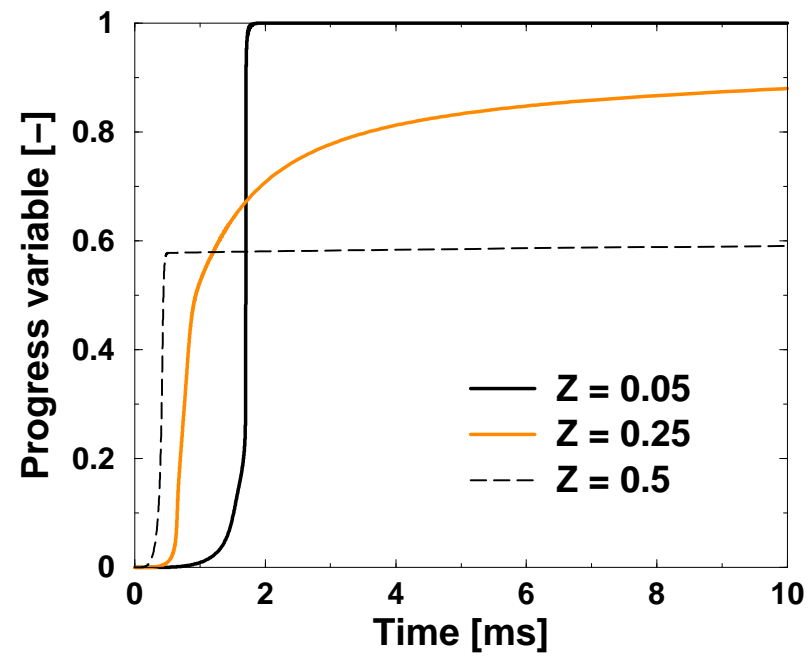

Figure 7. Evolution of the normalized progress variable for various mixture fractions for a homogeneous reactor configuration with $T^{\text {init }}=1100 K$. For $Z=0.25$, complete reaction $(c \rightarrow 1)$ is achieved near $t \sim 400 \mathrm{~ms}$. For $Z=0.5$, equilibrium is reached near $t \sim 200 \mu \mathrm{s}$. 


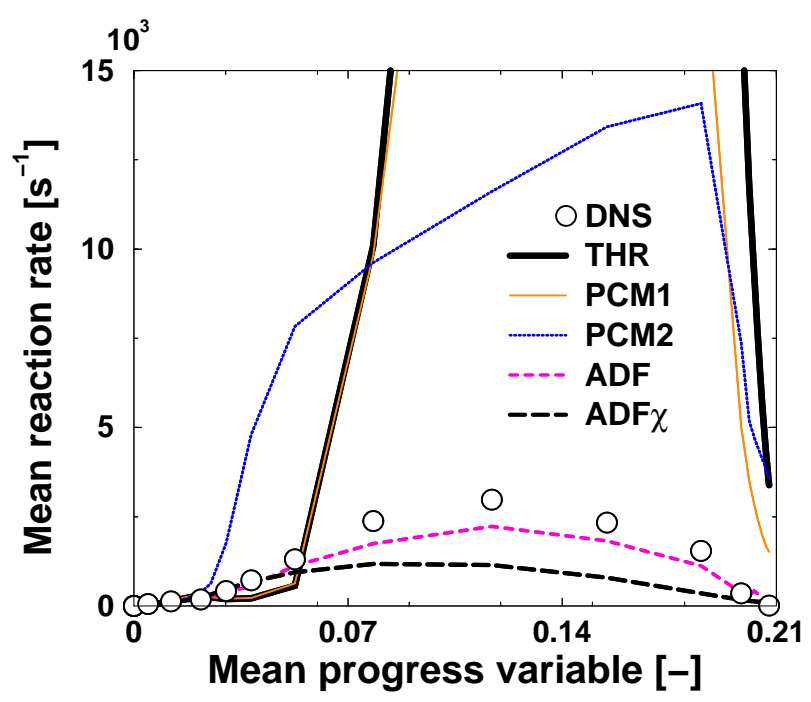

Figure 8. Evolution of the mean reaction rate of the progress variable $\left(\widetilde{\dot{\omega}_{Y_{c}}}\right)$ with the mean progress variable. Comparison between DNS results and tabulation models (Table 2). Case M-Sz06-Z01-Tu1100 (low $\widetilde{Z}$ ).

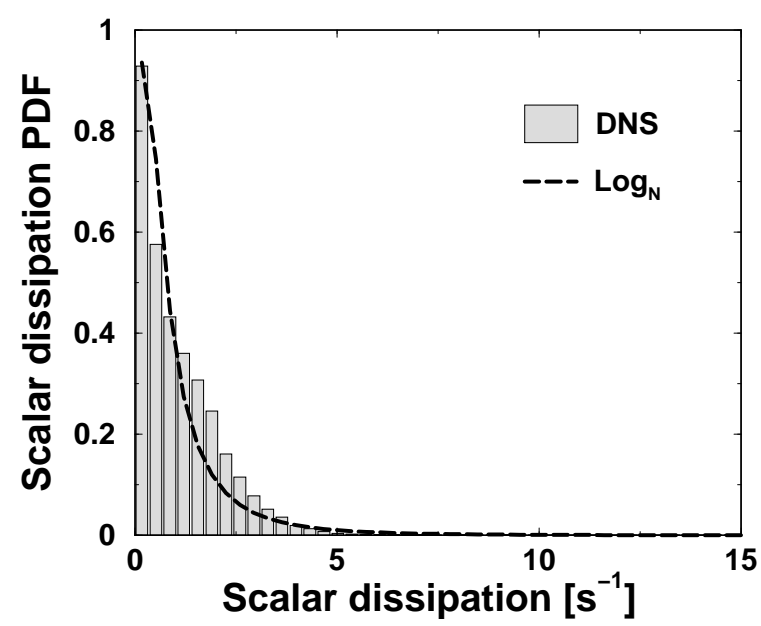

Figure 9. Probability density function of the scalar dissipation rate $\chi$. Comparison between DNS data distribution and the presumed log-normal PDF of the ADF $\chi$ model [19]. Case M-Sz06-Z01-Tu1100 (low $\widetilde{Z}$ ) at $\widetilde{Y_{c}}=0.12$. 


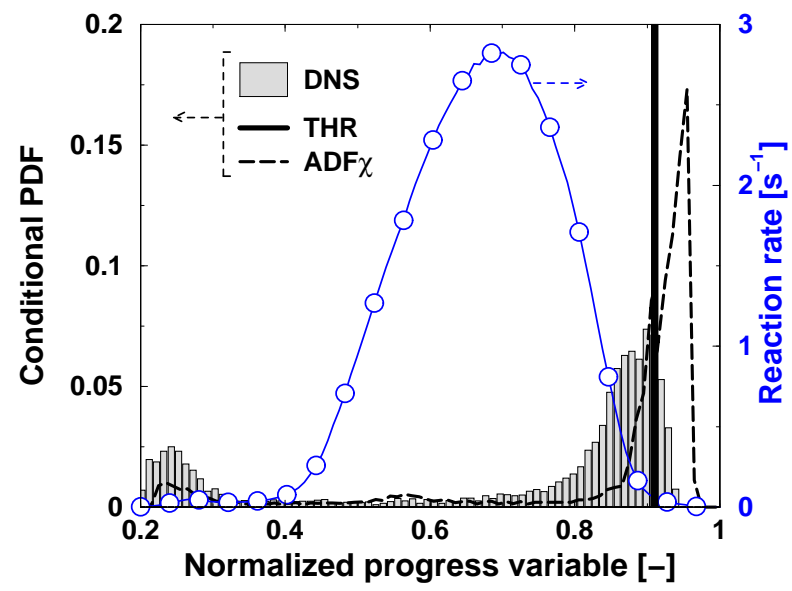

Figure 10. Conditional PDF of the progress variable $\bar{P}\left(c \mid Z^{*}\right)$ for $Z^{*}=0.13$ (most common mixture fraction in the heterogeneous reactor). The conditional reaction rate $\dot{\omega}_{H R}\left(c \mid Z^{*}\right)$ is also plotted. Case M-Sz06-Z01-Tu1100 (low $\widetilde{Z})$ at $\widetilde{Y_{c}}=0.12$.

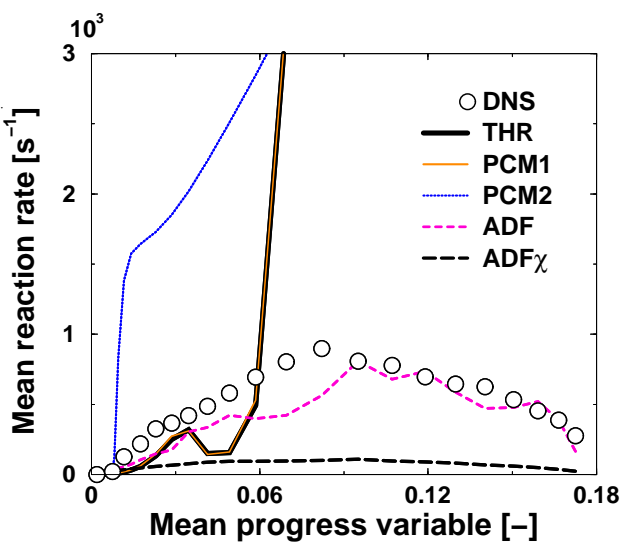

(a) Case T-Sz06-Z01-Tu950

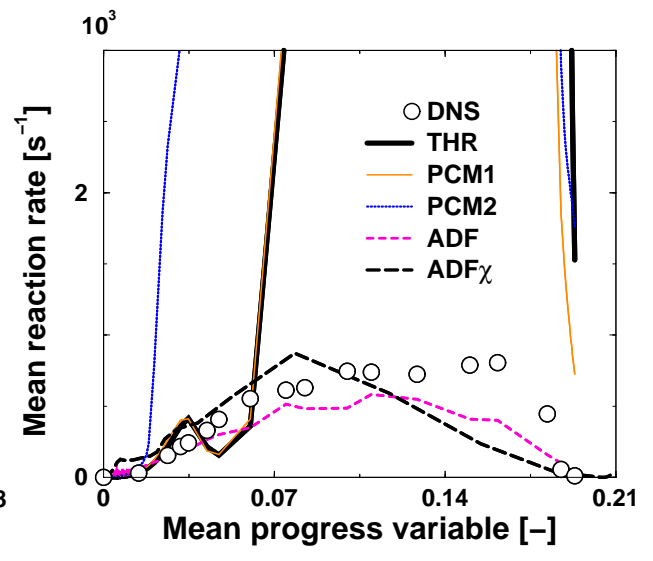

(b) Case T-Sz06-Z01-Tv650-950

Figure 11. Evolution of the mean reaction rate of the progress variable $\left(\widetilde{\dot{\omega}_{Y_{c}}}\right)$ with the mean progress variable. Comparison between DNS results and tabulation models (Table 2). Left: Case T-Sz06-Z01Tu950 (950 K is within NTC region). Right: Case T-Sz06-Z01-Tv650-950 (NTC range). 


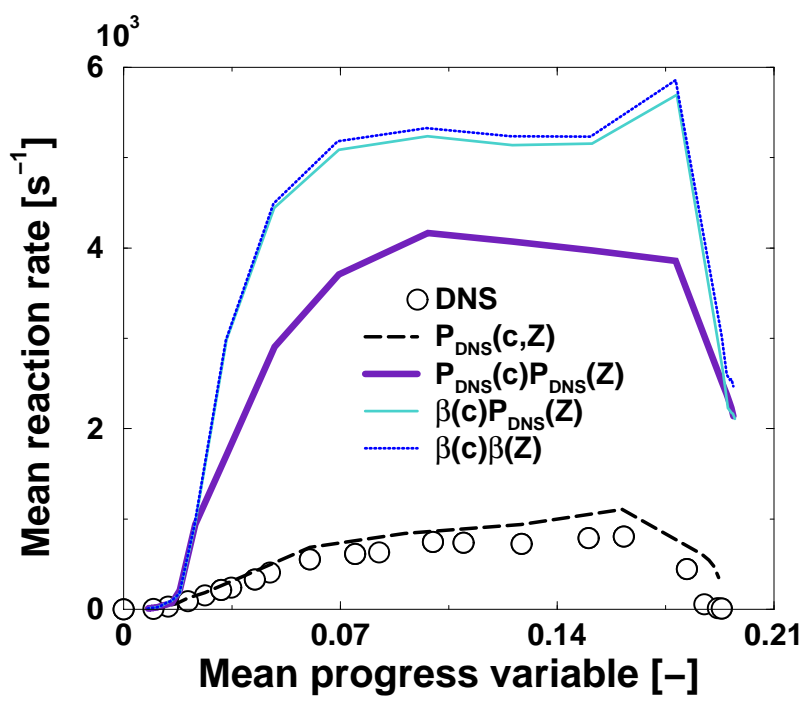

Figure 12. Influence of the PCM2 model hypothesis on the prediction of $\widetilde{\dot{\omega}_{Y_{c}}}$. Circles correspond to reference DNS results. $P_{\mathrm{DNS}}(c, Z)$ corresponds to Equation (7). $P_{\mathrm{DNS}}(c) P_{\mathrm{DNS}}(Z)$ corresponds to $Z$ and $c$ independence (Hypothesis 2.1). $\beta(c) P_{\mathrm{DNS}}(Z)$ adds Hypothesis 2.3 , and $\beta(c) \beta(Z)$ is the complete PCM2 model with the addition of Hypothesis 2.2.

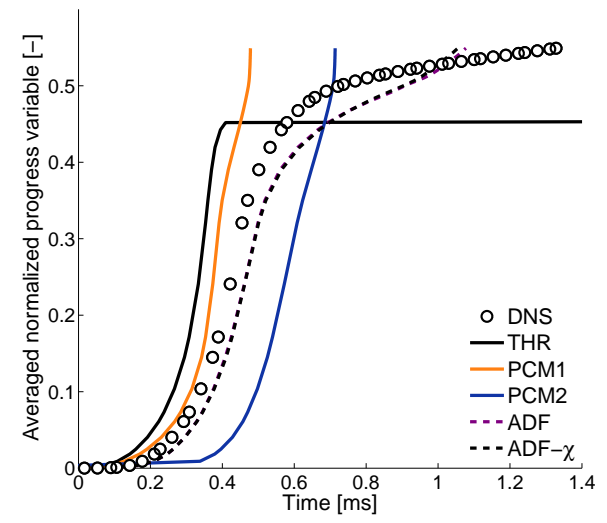

(a) M-Sz06-Z05-Tu1100

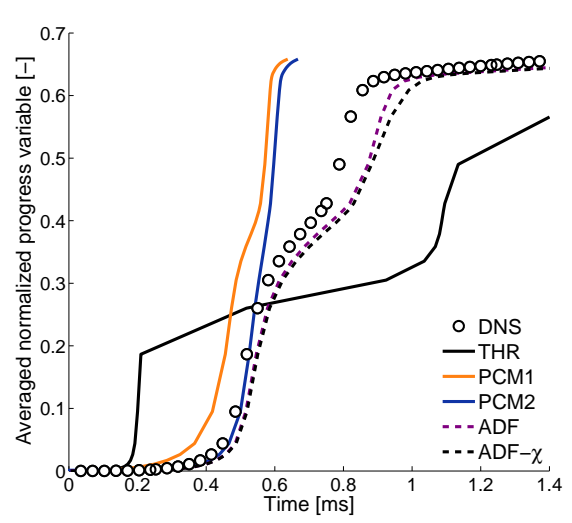

(b) M-Sz06-Z025-Tu1100

Figure 13. Averaged progress variable evolution for DNS and models 


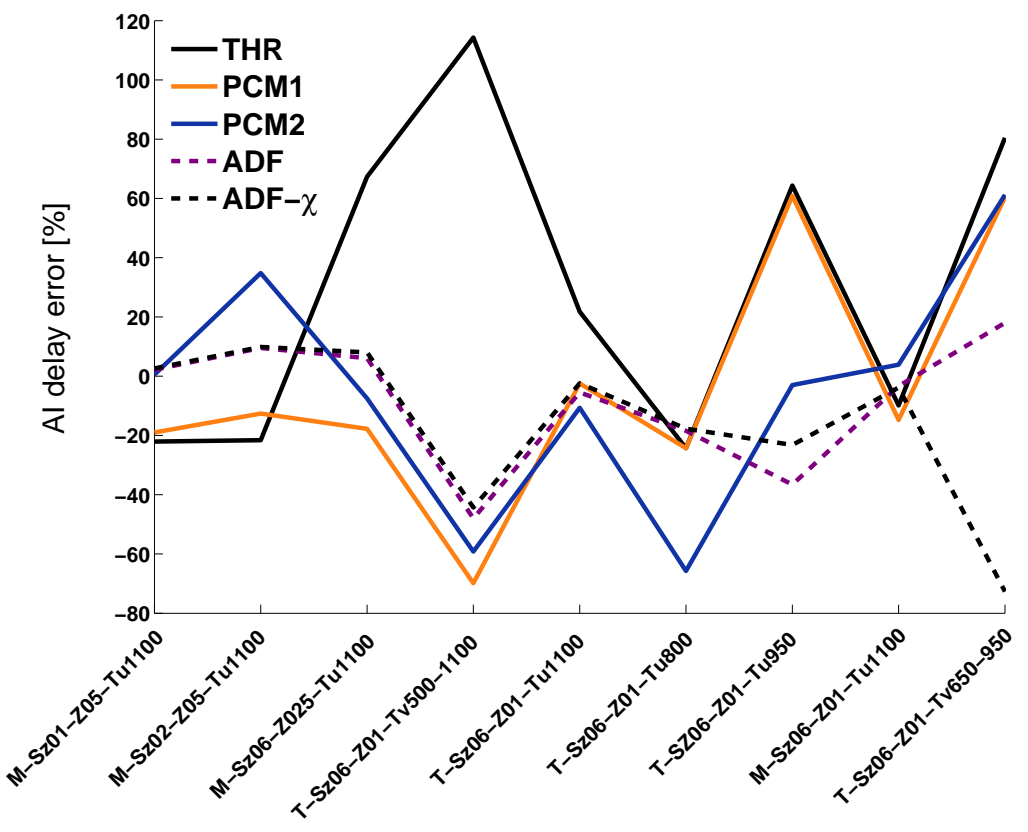

Figure 14. Relative error between models and DNS for the AI-Delay 